\title{
Warm versus cold cardioplegia in cardiac surgery: A meta-analysis with trial sequential analysis
}

Thompson Ka Ming Kot, MBChB, ${ }^{\mathrm{a}, \mathrm{b}}$ Jeffrey Shi Kai Chan, MBChB, ${ }^{\mathrm{a}, \mathrm{c}}$ Saied Froghi, MRCS, ${ }^{\mathrm{d}}$ Dawnie Ho Hei Lau, MBChB, ${ }^{\mathrm{a}, \mathrm{c}}$ Kara Morgan, MPharm, MSc, PGHE, ${ }^{\mathrm{e}, \mathrm{f}}$ Francesco Magni, BSc, ${ }^{\mathrm{g}}$ and Amer Harky, MRCS, MSc ${ }^{\text {h }}$

\section{ABSTRACT}

Objective: This meta-analysis aimed to compare clinical outcomes of warm and cold cardioplegia in cardiac surgeries in adult patients, with trial sequential analysis (TSA) used to determine the conclusiveness of the results.

Methods: Electronic searches were performed on PubMed, Medline, Scopus, EMBASE, and Cochrane library to identify all studies that compared warm and cold cardioplegia in cardiac surgeries. Primary end points were in-hospital or 30-day mortality, myocardial infarction, low cardiac output syndrome, intra-aortic balloon pump use, stroke, and new atrial fibrillation. Secondary end points were acute kidney injury, hospital length of stay, and intensive care unit length of stay. Prespecified subgroup analyses were performed for (1) studies published since publication of Fan and colleagues in 2010, (2) randomized controlled studies, (3) studies with low risk of bias, (4) coronary artery bypass graft surgeries, and (5) studies with cold blood versus those with cold crystalloid cardioplegia. TSA was performed to determine conclusiveness of the results, using on all outcomes without significant heterogeneity from studies of low risk of bias.

Results: No significant differences were found between post-operative rates of mortality, myocardial infarction, low cardiac output syndrome, intra-aortic balloon pump use, stroke, new atrial fibrillation, and acute kidney injury between warm and cold cardioplegia. TSA concluded that current evidence was sufficient to rule out a $20 \%$ relative risk reduction in these outcomes.

Conclusions: Concerning safety outcomes, current evidence suggests that the choice between warm and cold cardioplegia remains in the surgeon's preference. (JTCVS Open 2021;6:161-90)

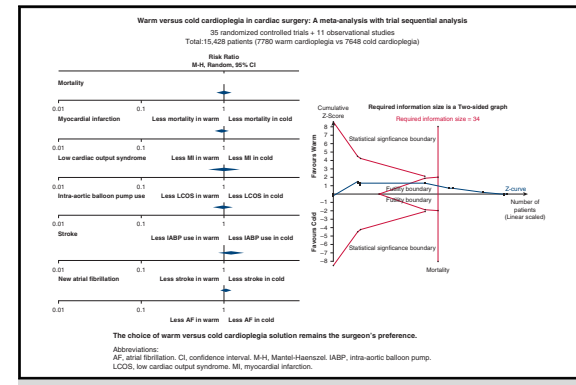

No significant differences were found in major postoperative outcomes between warm and cold cardioplegia. $\mathrm{M}-\mathrm{H}$, Mantel-Haenszel; $\mathrm{Cl}$, confidence interval.

\section{CENTRAL MESSAGE}

The choice between warm and cold cardioplegia remains the surgeon's preference.

\section{PERSPECTIVE}

This systematic review and meta-analysis showed no differences between postoperative rates of mortality, MI, LCOS, IABP use, stroke, new AF, and AKI between warm and cold cardioplegia. TSA concluded that current evidence was sufficient to rule out a $20 \%$ relative risk reduction in these outcomes.

See Commentary on page 191.
Cardioplegia allows for a still operative field, which is important in cardiac surgeries. There are various forms of cardioplegic solutions nowadays, which can be administrated in different ways. These include blood versus crystalloid, cold versus warm, intermittent versus continuous, antegrade versus retrograde versus combined, and terminal warm shot cardioplegia.

\footnotetext{
From the ${ }^{\mathrm{a}}$ Faculty of Medicine, The Chinese University of Hong Kong, Shatin, New Territories, Hong Kong; ' Department of Anaesthesia and Intensive Care, Prince of Wales Hospital, Shatin, New Territories, Hong Kong; ' Division of Cardiology, Department of Medicine and Therapeutics, Prince of Wales Hospital, Hong Kong; ${ }^{\mathrm{d} D i v i s i o n}$ of Surgery and Interventional Sciences, Royal Free Hospital, University College London, London; ${ }^{\mathrm{e}}$ Department of Cardiology, Manchester Royal Infirmary, Manchester, United Kingdom; ${ }^{\mathrm{f}}$ Faculty of Biology, Medicine \&amp; Health, Division of Pharmacy \&amp; Optometry, School of Health Sciences, The University of Manchester, Manchester, United Kingdom; ${ }^{\mathrm{g}}$ Faculty of Medicine, University College London, London, United Kingdom; and ${ }^{\mathrm{h}}$ Depart-
}

Concerns have long been raised about the clinical outcomes of different forms of cardioplegia. Since the 1970s, there has been debate over the optimal temperature for cardioplegic solutions. Cold cardioplegia has been used to maximize myocardial cooling and metabolic inhibition. In contrast, warm cardioplegia was proposed as an alternative to meet the energy demands of the arrested heart; lower the

ment of Cardiothoracic Surgery, Liverpool Heart and Chest Hospital, Liverpool, United Kingdom.

Received for publication March 17, 2021; accepted for publication March 17, 2021; available ahead of print April 28, 2021.

Address for reprints: Francesco Magni, University College London Medical School, London, United Kingdom (E-mail: franceso.magni.16@ucl.ac.uk). 2666-2736

Copyright (C 2021 The Author(s). Published by Elsevier Inc. on behalf of The American Association for Thoracic Surgery. This is an open access article under the CC BY license (http://creativecommons.org/licenses/by/4.0/).

https://doi.org/10.1016/j.xjon.2021.03.011 


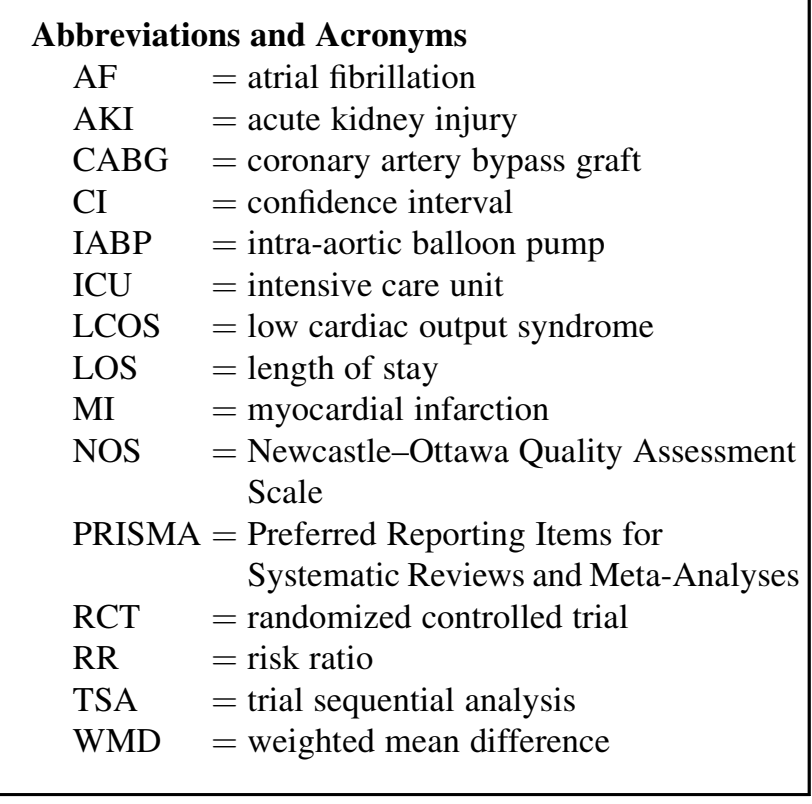

risk of membrane destabilization, intracellular edema, calcium sequestration, and time for heart rewarming; and decrease the risk of reperfusion injury. Besides, blood was considered to be better than crystalloid cardioplegia due to its greater oxygen-carrying and buffering capacity, better microvascular flow secondary to rheologic effects, and less associated intracellular edema. ${ }^{1}$

The Warm Heart Investigators ${ }^{2}$ conducted a randomized controlled trial (RCT) of 1732 patients in 1994. They demonstrated a significant reduction in postoperative low cardiac output syndrome (LCOS) in the warm cardioplegia group, without significant differences in 30-day all-cause mortality, postoperative myocardial infarction (MI), and stroke. A meta-analysis on RCTs by Fan and colleagues ${ }^{3}$ showed no significant difference in the clinical outcomes investigated. However, it was unclear whether the results were conclusive. This systemic review and meta-analysis aimed to compare clinical outcomes of warm versus cold cardioplegia in adult cardiac surgeries, updating the metaanalysis by Fan and colleagues ${ }^{3}$ with more recent evidence, further analyzing the conclusiveness of the results.

\section{METHODS}

This systematic review and meta-analysis was conducted according to the Preferred Reporting Items for Systematic Reviews and MetaAnalyses (PRISMA) statement and methods stipulated in the Cochrane Handbook for Systematic Review of Interventions. ${ }^{4,5}$ It has been submitted to PROSPERO with a registration number of CRD42020171613 but had not been approved as of the time of submission.

\section{Search Strategy and Selection Criteria}

Electronic searches were performed on PubMed, Medline, Scopus, EMBASE, and Cochrane library to identify all studies comparing warm and cold cardioplegia in cardiac surgeries regardless of publication type or language. All databases were searched since the search of previous metaanalysis (Fan and colleagues ${ }^{3}$ ) on the topic, up until June 27, 2020. A search was also conducted on ClinicalTrials. gov to identify ongoing or unpublished clinical trials. The search string used was ([warm OR normothermia OR normothermic OR cold OR hypothermia OR hypothermic] AND [cardioplegia OR "myocardial protection"] AND [valve OR valvular OR AVR OR MVR OR DVR OR TVR OR PVR OR "coronary artery bypass graft" OR "coronary artery bypass grafting" OR CABG OR "vein graft" OR "bypass graft" OR "surgical revascularization"]). All search terms searched as both key words and Medical Subject Headings terms to maximize sensitivity. Reference lists of papers found in the literature search were manually searched to assess suitability for inclusion in this review.

Three reviewers performed literature screening (T.K.M.K., J.S.K.C., Shaik Ashraf Bin Shaik Ismail). Articles were first screened based on their titles and abstracts. Full texts of all identified articles were then retrieved and systemically assessed using the inclusion and exclusion criteria for further study. Conflicts over inclusion were resolved by consensus. Articles were deemed eligible for inclusion if warm cardioplegia was compared against cold cardioplegia in cardiac surgeries. Noncomparative studies, conference abstracts or papers, articles involving fewer than 5 patients, and studies including patients younger than 18 years of age were excluded. Studies not reporting any of the end points specified herein were also excluded. Warm cardioplegia was defined as $28^{\circ} \mathrm{C}$ to $37^{\circ} \mathrm{C}$, whereas cold cardioplegia was defined as $4^{\circ} \mathrm{C}$ to $15^{\circ} \mathrm{C}$.

Primary end points were in-hospital or 30-day mortality, MI, LCOS, intra-aortic balloon pump (IABP) use, stroke, and new atrial fibrillation (AF). Secondary end points were acute kidney injury (AKI), hospital length of stay (LOS), and intensive care unit (ICU) LOS. Summary estimates were extracted manually from included studies. Only the most updated data were included wherever duplicate data existed. Study authors were contacted where necessary. Data reported by previous meta-analysis by Fan and colleagues in $2010^{3}$ were also extracted from published Forest plots. Conflicts over data extraction were resolved by consensus.

\section{Statistical Analysis}

All included studies were critically appraised by the modified Jadad scale for RCTs or the Newcastle-Ottawa Quality Assessment Scale (NOS) for observational studies. The modified Jadad scale is a numeral scale with components addressing randomization, blinding, selection, adverse effects assessment, and statistical methods. It is described in detail in Table E1. The NOS assessed cohort studies according to selection, comparability, and outcome and is detailed in Table E2. The metaanalysis by Fan and colleagues in $2010^{3}$ was critically appraised by the AMSTAR 2, which is a critical appraisal tool for systematic reviews that includes randomized or nonrandomized studies of health care interventions. $^{6}$

All statistical analyses were a priori, specified before the start of data extraction. Odds ratios and $95 \%$ confidence intervals (CIs) or weighted mean differences (WMDs) and $95 \%$ CIs were used as the main summary measures for baseline characteristics, whereas relative risks (RRs) and $95 \%$ CIs or WMD and $95 \%$ CIs were used as main summary measures for the outcomes studied. Discrete variables were pooled using the Mantel-Haenszel method with RR as the effect measure. Continuous variables were pooled using the inverse variance method with WMD as the effect measure. Sensitivity analysis is performed by the leave-one-out method. Prespecified subgroup analysis was performed on (1) studies published since publication of Fan and colleagues in $2010^{3}$; (2) RCTs; (3) studies with low risk of bias, defined by 5 or 7 score or more in modified Jadad scale or NOS, respectively; (4) coronary artery bypass graft (CABG) surgeries; and (5) studies with cold blood versus those with cold crystalloid cardioplegia.

Heterogeneity was assessed by the Cochran's Q test and $I^{2}$ statistics. All variables were analyzed using the DerSimonian-Laird random effects 
model. For variables reported by at least 10 studies, publication bias was assessed visually by funnel plot.

Trial sequential analysis (TSA) can be used to assess conclusiveness of meta-analytical findings. As evidence accumulates, random errors also accumulates and they may incidentally lead to "significant" results reported in meta-analysis. Meta-analyses of cardiovascular and anesthesiologic interventions have many false positions and negative results due to the low statistical power of the meta-analysis when the required number of participants or trials has not been reached, which can be addressed by TSA. ${ }^{7}$ Trials were included in chronological order and handled as interim analysis relative to the required information size, which is defined as the number of participants and events necessary to detect or reject an a priori assumed intervention effect in meta-analysis. Statistical techniques were used to adjust the CI of point estimate and to increase the threshold for statistical significance based on effect to be observed, incidence of outcome in control arm, information size, and heterogeneity. ${ }^{8}$ It was performed on all outcomes without significant heterogeneity, from studies of low risk of bias. Z-score curve was generated by plotting cumulative $\mathrm{Z}$ scores with new study data. A Z-score curve crossing either of statistical significance boundaries (ie, the pair of outer oblique lines) implies that the statistically significant data is conclusive, whereas crossing either of the futility boundaries (ie, inner oblique lines) implies that the statistically insignificant data is conclusive. If the curve crosses the required information size boundary (ie, the vertical line), all observations are said to be conclusive. $^{8}$ All available statistical information (Fisher information) was used. The Z-score threshold was adjusted using the O'Brien-Fleming alpha-spending function. Studies reporting no events were handled by adding a constant (1) to both arms. Required information sizes were estimated from an RR reduction of $20 \%$, chosen to represent a clinically meaningful effect. Incidences were calculated from all studies reporting the outcome of interest. Heterogeneity and variance adjustments were estimated from all included studies in TSA. A prespecified permissible 2 -sided type 1 error $(\alpha)$ of $5 \%$ and type 2 error $(\beta)$ of $20 \%$ were used, therefore giving a power of $80 \%$.

All $P$ values are 2 -sided. The meta-analytical component was performed using Review Manager (RevMan), version 5.3 (Copenhagen: The Nordic Cochrane Centre, The Cochrane Collaboration, 2014). The TSA component was performed using the Copenhagen trial unit, TSA software, version 0.9.5.10 Beta.

\section{RESULTS}

The literature search is summarized in a PRISMA diagram (Figure 1). A total of 2802 nonduplicate citations

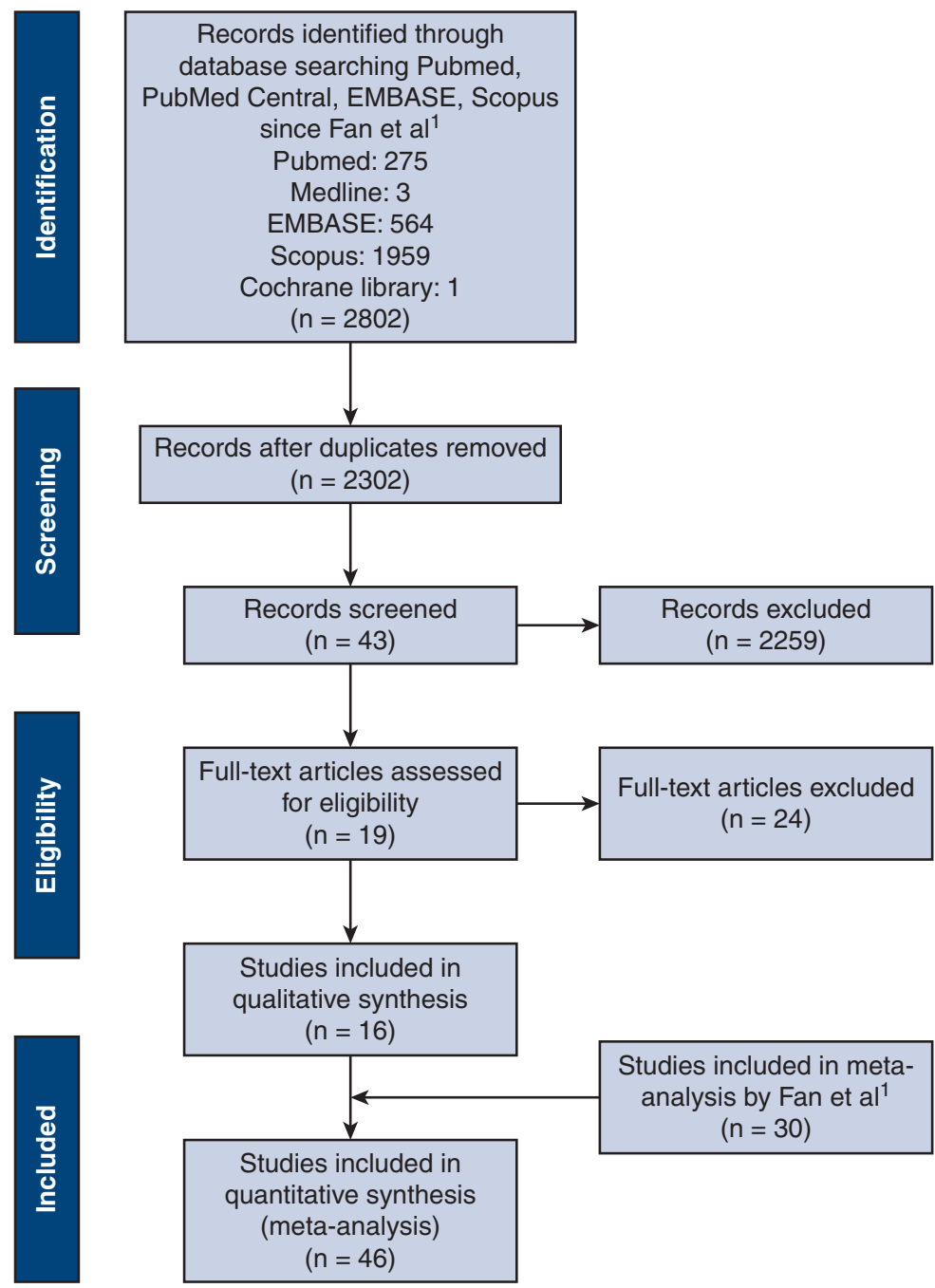

FIGURE 1. The Preferred Reporting Items for Systematic Reviews and Meta-Analyses (PRISMA) flow diagram. 
TABLE 1. Characteristics of studies included

\begin{tabular}{|c|c|c|c|c|c|c|c|c|}
\hline Author & Year & $\begin{array}{l}\text { Surgery } \\
\text { type }\end{array}$ & $\begin{array}{l}\text { No. of } \\
\text { patients } \\
\text { (warm } \\
\text { cohort) }\end{array}$ & $\begin{array}{l}\text { No. of } \\
\text { patients } \\
\text { (cold } \\
\text { cohort) }\end{array}$ & $\begin{array}{c}\text { Warm } \\
\text { cardioplegia } \\
\text { temperature, }{ }^{\circ} \mathrm{C}\end{array}$ & $\begin{array}{c}\text { Cold } \\
\text { cardioplegia } \\
\text { temperature, }{ }^{\circ} \mathrm{C}\end{array}$ & Key finding & $\begin{array}{l}\text { Risk of } \\
\text { bias -MJS } \\
\text { (/8) NOS } \\
\text { (/9) }\end{array}$ \\
\hline Ali et $\mathrm{al}^{\mathrm{E} 2}$ & 1994 & CABG, valve & 38 & CB: 38 & 37 & 10 & $\begin{array}{l}\text { Intermittent warm blood was as } \\
\text { safe as cold blood cardioplegia } \\
\text { when the aortic crossclamp } \\
\text { time was less than } 90 \text { min. }\end{array}$ & $3 / 8$ \\
\hline Ascione et $\mathrm{al}^{\mathrm{E} 3}$ & 2002 & Valve & 19 & CB: 16 & 34 & $6-8$ & $\begin{array}{l}\text { Warm blood cardioplegia was } \\
\text { associated with more ischemic } \\
\text { stress and myocardial injury, as } \\
\text { compared with cold blood } \\
\text { cardioplegia in patients with } \\
\text { aortic stenosis undergoing } \\
\text { valvular replacement. }\end{array}$ & $5 / 8$ \\
\hline Raza Baig et al ${ }^{\mathrm{E} 37}$ & 2015 & CABG & 94 & CB: 121 & NR & NR & $\begin{array}{l}\text { Intermittent antegrade warm } \\
\text { blood cardioplegia was } \\
\text { associated with better } \\
\text { myocardial protection in early } \\
\text { postoperative period. }\end{array}$ & $9 / 9$ \\
\hline Baron et al ${ }^{\mathrm{E} 4}$ & 2003 & CABG & 48 & CB: 21 & 37 & 15 & $\begin{array}{l}\text { Warm and cold blood } \\
\text { cardioplegia were comparable } \\
\text { in terms of postoperative } \\
\text { complications and mortality } \\
\text { rate. }\end{array}$ & $3 / 8$ \\
\hline Candilio et al ${ }^{\mathrm{E} 38}$ & 2014 & CABG & 10 & CB: 28 & NR & NR & $\begin{array}{l}\text { Antegrade retrograde } \\
\text { cardioplegia was associated } \\
\text { with less perioperative } \\
\text { myocardial infarction } \\
\text { compared with antegrade } \\
\text { cardioplegia. }\end{array}$ & $9 / 9$ \\
\hline Chello et $\mathrm{al}^{\mathrm{E} 5}$ & 1997 & CABG & 20 & CB: 20 & 37 & 5 & $\begin{array}{l}\text { Warm cardioplegia was } \\
\text { associated with increased } \\
\text { activation of complement and } \\
\text { neutrophils compared with cold } \\
\text { cardioplegia. }\end{array}$ & $2 / 8$ \\
\hline Chello et $\mathrm{al}^{\mathrm{E} 6}$ & 2003 & CABG & 20 & CB: 20 & 37 & 5 & $\begin{array}{l}\text { Intermittent warm cardioplegia } \\
\text { was associated with better } \\
\text { myocardial protection, and } \\
\text { increased HSP72 expression. }\end{array}$ & $4 / 8$ \\
\hline Chocron et $\mathrm{al}^{\mathrm{E} 7}$ & 2000 & CABG & 45 & $\mathrm{CB}: 45$ & 37 & 8 & $\begin{array}{l}\text { Intermittent warm blood } \\
\text { cardioplegia was associated } \\
\text { with comparable postoperative } \\
\text { complications and fewer } \\
\text { myocardial injuries in low-risk } \\
\text { patients. }\end{array}$ & $6 / 8$ \\
\hline Curtis et al ${ }^{\mathrm{E}}$ & 1996 & CABG & 40 & CB: 38 & NR & 4 & $\begin{array}{l}\text { Warm cardioplegia was } \\
\text { associated with comparable } \\
\text { morbidity and mortality } \\
\text { compared with cold } \\
\text { cardioplegia. }\end{array}$ & $4 / 8$ \\
\hline Dar et $\mathrm{al}^{\mathrm{E} 9}$ & 2005 & CABG & 20 & $\mathrm{CC}: 10$ & 37 & 4 & $\begin{array}{l}\text { Antegrade with retrograde warm } \\
\text { blood cardioplegia was }\end{array}$ & $4 / 8$ \\
\hline
\end{tabular}


TABLE 1. Continued

\begin{tabular}{|c|c|c|c|c|c|c|c|c|}
\hline Author & Year & $\begin{array}{c}\text { Surgery } \\
\text { type }\end{array}$ & $\begin{array}{l}\text { No. of } \\
\text { patients } \\
\text { (warm } \\
\text { cohort) }\end{array}$ & $\begin{array}{l}\text { No. of } \\
\text { patients } \\
\text { (cold } \\
\text { cohort) }\end{array}$ & $\begin{array}{c}\text { Warm } \\
\text { cardioplegia } \\
\text { temperature, }{ }^{\circ} \mathrm{C}\end{array}$ & $\begin{array}{c}\text { Cold } \\
\text { cardioplegia } \\
\text { temperature, }{ }^{\circ} \mathrm{C}\end{array}$ & Key finding & $\begin{array}{l}\text { Risk of } \\
\text { bias -MJS } \\
\text { (/8) NOS } \\
\text { (/9) }\end{array}$ \\
\hline & & & & & & & $\begin{array}{l}\text { associated with lower } \\
\text { postoperative cardiac enzymes } \\
\text { compared with antegrade } \\
\text { cardioplegia. }\end{array}$ & \\
\hline De Jonge et $\mathrm{al}^{\mathrm{E} 39}$ & 2015 & CABG & 2585 & CC: 2585 & 37 & 4 & $\begin{array}{l}\text { Blood cardioplegia was an } \\
\text { independent risk factor for } \\
\text { increased creatine kinase-MB } \\
\text { after CABG. }\end{array}$ & $8 / 9$ \\
\hline Elwatidy et $\mathrm{al}^{\mathrm{E} 10}$ & 1999 & CABG & 47 & $\begin{array}{c}\text { CB: } 40 \\
\text { CC: } 41\end{array}$ & $28-30$ & $\begin{array}{l}\text { CB: } 8 \\
\text { CC: } 4\end{array}$ & $\begin{array}{l}\text { Warm blood cardioplegia was } \\
\text { associated with better } \\
\text { metabolic and functional } \\
\text { recovery, without significant } \\
\text { differences in morbidity and } \\
\text { mortality. }\end{array}$ & $4 / 8$ \\
\hline Engelman et $\mathrm{al}^{\mathrm{E} 11}$ & 1996 & CABG & 93 & CB: 37 & $32 / 37$ & $8-10$ & $\begin{array}{l}\text { Warm cardioplegia was } \\
\text { associated with more activation } \\
\text { of fibrinolytic potential and } \\
\text { fewer neurologic adverse } \\
\text { events. }\end{array}$ & $8 / 8$ \\
\hline Franke et $\mathrm{al}^{\mathrm{E} 12}$ & 2003 & CABG & 100 & CB: 100 & 33 & 4 & $\begin{array}{l}\text { Intermittent antegrade warm } \\
\text { blood cardioplegia was } \\
\text { associated with lower } \\
\text { postoperative cardiac enzymes. }\end{array}$ & $6 / 8$ \\
\hline Gaudino et $\mathrm{al}^{\mathrm{E} 13}$ & 2013 & Valve & 29 & CC: 31 & 37 & 0 & $\begin{array}{l}\text { Warm cardioplegia was } \\
\text { associated with better right } \\
\text { ventricular protection } \\
\text { compared with one-shot } \\
\text { histidine-tryptophane- } \\
\text { ketoglutarate cardioplegia } \\
\text { solution. }\end{array}$ & $7 / 8$ \\
\hline Hayashida et al ${ }^{\mathrm{E} 14}$ & 1994 & CABG & 48 & CB: 24 & $\begin{array}{l}\text { W: } 37 \\
\text { L: } 29\end{array}$ & 8 & $\begin{array}{l}\text { Warm cardioplegia was } \\
\text { associated with more lactate } \\
\text { and acid washout with } \\
\text { reperfusion and better cardiac } \\
\text { function postoperatively. }\end{array}$ & $4 / 8$ \\
\hline Hayashida et al ${ }^{\mathrm{E} 15}$ & 1995 & CABG & 28 & CB: 14 & $\begin{array}{l}\text { W: } 37 \\
\text { L: } 29\end{array}$ & 9 & $\begin{array}{l}\text { Warm and tepid cardioplegia were } \\
\text { associated with better cardiac } \\
\text { function postoperatively. }\end{array}$ & $4 / 8$ \\
\hline Honkonen et $\mathrm{al}^{\mathrm{E} 16}$ & 1997 & CABG & 15 & CB: 14 & 37 & $5-7$ & $\begin{array}{l}\text { Warm cardioplegia was } \\
\text { associated with better recovery } \\
\text { of right ventricular function in } \\
\text { terms of ejection fraction and } \\
\text { preload related stroke work and } \\
\text { less postoperative cardiac } \\
\text { enzymes release. }\end{array}$ & $4 / 8$ \\
\hline 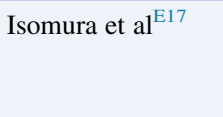 & 1995 & CABG & 29 & CC: 26 & $26-37$ & 4 & $\begin{array}{l}\text { Warm cardioplegia was } \\
\text { associated with comparable } \\
\text { myocardial protection and }\end{array}$ & $3 / 8$ \\
\hline
\end{tabular}


TABLE 1. Continued

\begin{tabular}{|c|c|c|c|c|c|c|c|c|}
\hline Author & Year & $\begin{array}{c}\text { Surgery } \\
\text { type }\end{array}$ & $\begin{array}{c}\text { No. of } \\
\text { patients } \\
\text { (warm } \\
\text { cohort) }\end{array}$ & $\begin{array}{l}\text { No. of } \\
\text { patients } \\
\text { (cold } \\
\text { cohort) }\end{array}$ & $\begin{array}{c}\text { Warm } \\
\text { cardioplegia } \\
\text { temperature, }{ }^{\circ} \mathrm{C}\end{array}$ & $\begin{array}{c}\text { Cold } \\
\text { cardioplegia } \\
\text { temperature, }{ }^{\circ} \mathrm{C}\end{array}$ & Key finding & $\begin{array}{c}\text { Risk of } \\
\text { bias -MJS } \\
\text { (/8) NOS } \\
\text { (/9) }\end{array}$ \\
\hline & & & & & & & $\begin{array}{l}\text { clinical outcomes compared } \\
\text { with cold cardioplegia. }\end{array}$ & \\
\hline 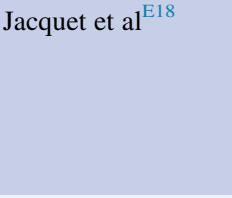 & 1999 & CABG & 108 & CC: 92 & 37 & NR & $\begin{array}{l}\text { Intermittent antegrade warm } \\
\text { blood cardioplegia was } \\
\text { associated with lower } \\
\text { postoperative cardiac enzyme } \\
\text { release. }\end{array}$ & $5 / 8$ \\
\hline Kammerer et $\mathrm{al}^{\mathrm{E} 19}$ & 2010 & Valve & 52 & CC: 55 & 35 & 4 & $\begin{array}{l}\text { Warm blood cardioplegia was } \\
\text { associated with significantly } \\
\text { greater mortality rate compared } \\
\text { with cold crystalloid } \\
\text { cardioplegia. }\end{array}$ & $4 / 8$ \\
\hline Kuhn et $\mathrm{al}^{\mathrm{E} 20}$ & 2015 & CABG & 36 & CB: 32 & 37 & $4-6$ & $\begin{array}{l}\text { Intermittent warm cardioplegia } \\
\text { was associated with greater } \\
\text { extent of endothelial injury and } \\
\text { comparable rates of clinical } \\
\text { end points compared with cold } \\
\text { cardioplegia. }\end{array}$ & $7 / 8$ \\
\hline Kuhn et $\mathrm{al}^{\mathrm{E} 40}$ & 2018 & CABG & 212 & CB: 212 & 37 & $4-6$ & $\begin{array}{l}\text { No significant differences were } \\
\text { found in myocardial protection } \\
\text { and similar postoperative } \\
\text { adverse events between } \\
\text { Buckberg and Calafiore } \\
\text { cardioplegia. }\end{array}$ & $8 / 9$ \\
\hline Lajos et al ${ }^{\mathrm{E} 21}$ & 1993 & CABG & 54 & $\begin{array}{l}\text { CB: } 54 \\
\text { CB: } 55\end{array}$ & 37 & NR & $\begin{array}{l}\text { Intermittent cold cardioplegia } \\
\text { provided a clearer operative } \\
\text { field compared with continuous } \\
\text { warm cardioplegia, without } \\
\text { better myocardial protection. }\end{array}$ & $3 / 8$ \\
\hline Landymore et $\mathrm{al}^{\mathrm{E} 22}$ & 1996 & CABG & 20 & CB: 20 & 37 & 8 & $\begin{array}{l}\text { Warm cardioplegia was } \\
\text { associated with comparable } \\
\text { myocardial metabolic and } \\
\text { functional recovery and } \\
\text { postoperative adverse events } \\
\text { compared with cold } \\
\text { cardioplegia. }\end{array}$ & $5 / 8$ \\
\hline Maccherini et $\mathrm{al}^{\mathrm{E} 23}$ & 1995 & CABG & 50 & $\mathrm{CB}: 50$ & 37 & $4-8$ & $\begin{array}{l}\text { Warm blood cardioplegia was } \\
\text { associated with less pleural } \\
\text { effusions and thoracentesis } \\
\text { related to hypothermia. }\end{array}$ & $2 / 8$ \\
\hline Martin et al ${ }^{\mathrm{E} 24}$ & 1994 & CABG & 493 & CC: 508 & $\geq 35$ & $\leq 8$ & $\begin{array}{l}\text { Warm cardioplegia was } \\
\text { associated with more } \\
\text { neurologic events, as defined as } \\
\text { stroke and encephalopathy, } \\
\text { compared with cold } \\
\text { cardioplegia. }\end{array}$ & $4 / 8$ \\
\hline Mourad et al ${ }^{\mathrm{E} 41}$ & 2016 & CABG & 50 & CC: 50 & NR & NR & $\begin{array}{l}\text { Antegrade warm blood } \\
\text { cardioplegia was associated }\end{array}$ & $9 / 9$ \\
\hline
\end{tabular}


TABLE 1. Continued

\begin{tabular}{|c|c|c|c|c|c|c|c|c|}
\hline Author & Year & $\begin{array}{c}\text { Surgery } \\
\text { type }\end{array}$ & $\begin{array}{l}\text { No. of } \\
\text { patients } \\
\text { (warm } \\
\text { cohort) }\end{array}$ & $\begin{array}{l}\text { No. of } \\
\text { patients } \\
\text { (cold } \\
\text { cohort) }\end{array}$ & $\begin{array}{c}\text { Warm } \\
\text { cardioplegia } \\
\text { temperature, }{ }^{\circ} \mathrm{C}\end{array}$ & $\begin{array}{c}\text { Cold } \\
\text { cardioplegia } \\
\text { temperature, }{ }^{\circ} \mathrm{C}\end{array}$ & Key finding & $\begin{array}{l}\text { Risk of } \\
\text { bias -MJS } \\
\text { (/8) NOS } \\
\text { (/9) }\end{array}$ \\
\hline & & & & & & & $\begin{array}{l}\text { with lower postoperative } \\
\text { cardiac enzymes release. }\end{array}$ & \\
\hline Nardi et $\mathrm{al}^{\mathrm{E} 42}$ & 2018 & $\begin{array}{l}\text { CABG } \\
\text { Valve }\end{array}$ & 159 & CC: 32 & $35-36$ & 4 & $\begin{array}{l}\text { Cold crystalloid cardioplegia was } \\
\text { associated with less } \\
\text { postoperative cardiac enzymes } \\
\text { release and comparable } \\
\text { postoperative clinical } \\
\text { outcomes compared with warm } \\
\text { blood cardioplegia. }\end{array}$ & $8 / 9$ \\
\hline Nardi et $\mathrm{al}^{\mathrm{E} 43}$ & 2018 & CABG & 297 & CC: 33 & $34-35$ & 4 & $\begin{array}{l}\text { No significant differences were } \\
\text { found in postoperative clinical } \\
\text { outcomes between warm and } \\
\text { cold cardioplegia in patients } \\
\text { undergoing CABG. }\end{array}$ & $8 / 9$ \\
\hline Pelletier et al ${ }^{\mathrm{E} 25}$ & 1994 & CABG & 100 & CB: 100 & NR & NR & $\begin{array}{l}\text { Warm cardioplegia was } \\
\text { associated with less } \\
\text { postoperative cardiac enzymes } \\
\text { release, and comparable rates } \\
\text { of mortality and myocardial } \\
\text { infarction compared with cold } \\
\text { cardioplegia. }\end{array}$ & $6.5 / 8$ \\
\hline Pepper et al ${ }^{\mathrm{E} 26}$ & 1995 & Valve & 15 & $\begin{array}{l}\text { CB: } 17 \\
\text { CC: } 15\end{array}$ & 37 & 4 & $\begin{array}{l}\text { Blood cardioplegia was } \\
\text { associated with greater thiol } \\
\text { level. }\end{array}$ & $3.5 / 8$ \\
\hline Plicner et $\mathrm{al}^{\mathrm{E} 44}$ & 2017 & CABG & 124 & CC: 114 & 37 & 4 & $\begin{array}{l}\text { No significant differences were } \\
\text { found in postoperative } \\
\text { systemic inflammatory } \\
\text { response and oxidative stress, } \\
\text { between warm and cold } \\
\text { cardioplegia. }\end{array}$ & $9 / 9$ \\
\hline Rashid et al $^{\mathrm{E} 27}$ & 1994 & CABG & 137 & CB: 144 & 37 & $4-6$ & $\begin{array}{l}\text { No significant differences were } \\
\text { found between warm and cold } \\
\text { cardioplegia for myocardial } \\
\text { protection and postoperative } \\
\text { adverse clinical outcomes. }\end{array}$ & $2 / 8$ \\
\hline Rashid et $\mathrm{al}^{\mathrm{E} 28}$ & 1995 & CABG & 58 & $\mathrm{CB}: 50$ & 37 & 8 & $\begin{array}{l}\text { Warm cardioplegia was } \\
\text { associated with comparable } \\
\text { myocardial protection in } \\
\text { patients with left ventricular } \\
\text { dysfunction in CABG } \\
\text { compared with cold } \\
\text { cardioplegia. }\end{array}$ & $2 / 8$ \\
\hline Rosu et $\mathrm{al}^{\mathrm{E} 45}$ & 2012 & CABG & 54 & CB: 84 & 27.6 & 10.1 & $\begin{array}{l}\text { Tepid cardioplegia was associated } \\
\text { with a greater rate of LCOS } \\
\text { compared with cold } \\
\text { cardioplegia. }\end{array}$ & $8 / 9$ \\
\hline Sacl 1 et $\mathrm{al}^{\mathrm{E} 29}$ & 2019 & CABG & 20 & CB: 28 & 28.4 & 13.7 & $\begin{array}{l}\text { Cold cardioplegia was associated } \\
\text { with less myocardial injury and } \\
\text { postoperative morbidity }\end{array}$ & $3 / 8$ \\
\hline
\end{tabular}


TABLE 1. Continued

\begin{tabular}{|c|c|c|c|c|c|c|c|c|}
\hline Author & Year & $\begin{array}{c}\text { Surgery } \\
\text { type }\end{array}$ & $\begin{array}{c}\text { No. of } \\
\text { patients } \\
\text { (warm } \\
\text { cohort) }\end{array}$ & $\begin{array}{l}\text { No. of } \\
\text { patients } \\
\text { (cold } \\
\text { cohort) }\end{array}$ & $\begin{array}{c}\text { Warm } \\
\text { cardioplegia } \\
\text { temperature, }{ }^{\circ} \mathrm{C}\end{array}$ & $\begin{array}{c}\text { Cold } \\
\text { cardioplegia } \\
\text { temperature, }{ }^{\circ} \mathrm{C}\end{array}$ & Key finding & $\begin{array}{l}\text { Risk of } \\
\text { bias -MJS } \\
\text { (/8) NOS } \\
\text { (/9) }\end{array}$ \\
\hline & & & & & & & $\begin{array}{l}\text { compared with warm } \\
\text { cardioplegia. }\end{array}$ & \\
\hline Şirlak et $\mathrm{al}^{\mathrm{E} 30}$ & 2003 & CABG & 50 & CC: 50 & $32-34$ & $4-6$ & $\begin{array}{l}\text { No significant differences were } \\
\text { found in postoperative cardiac } \\
\text { enzymes release between tepid } \\
\text { and cold cardioplegia. }\end{array}$ & $5 / 8$ \\
\hline Sirvinskas et $\mathrm{al}^{\mathrm{E} 31}$ & 2005 & CABG & 101 & CC: 55 & $\begin{array}{r}\text { W: } 37 \\
\text { L: } 28-30\end{array}$ & 4 & $\begin{array}{l}\text { Intermittent antegrade warm } \\
\text { cardioplegia was associated } \\
\text { with lower postoperative } \\
\text { troponin T release, shorter } \\
\text { duration of tracheal intubation, } \\
\text { and hospital stay. }\end{array}$ & $6 / 8$ \\
\hline $\begin{array}{l}\text { The Warm Heart } \\
\text { Investigator }^{\mathrm{E} 32}\end{array}$ & 1994 & CABG & 860 & CB: 872 & 37 & $5-8$ & $\begin{array}{l}\text { Warm cardioplegia was } \\
\text { associated with significantly } \\
\text { lower rates of LCOS and } \\
\text { comparable rates of mortality, } \\
\text { stroke, and myocardial } \\
\text { infarction compared with cold } \\
\text { cardioplegia. }\end{array}$ & $6.5 / 8$ \\
\hline Trescher et al ${ }^{\mathrm{E} 46}$ & 2017 & $\begin{array}{l}\text { CABG } \\
\text { Valve }\end{array}$ & 610 & CB: 1578 & $32-34$ & $6-8$ & $\begin{array}{l}\text { No significant differences were } \\
\text { found in myocardial protection } \\
\text { between intermittent warm and } \\
\text { cold blood cardioplegia. }\end{array}$ & $8 / 9$ \\
\hline Ucak et $\mathrm{al}^{\mathrm{E} 33}$ & 2019 & CABG & 185 & CC: 112 & $33-34$ & 4 & $\begin{array}{l}\text { No significant differences were } \\
\text { found in clinical outcomes } \\
\text { between intermittent warm and } \\
\text { cold cardioplegia. }\end{array}$ & $5 / 8$ \\
\hline Yau et $\mathrm{al}^{\mathrm{E} 34}$ & 1992 & CABG & 48 & $\mathrm{CB}: 26$ & 37 & 5 & $\begin{array}{l}\text { No significant differences were } \\
\text { found in clinical outcomes } \\
\text { between warm and cold } \\
\text { cardioplegia. }\end{array}$ & $3 / 8$ \\
\hline Yau et $\mathrm{al}^{\mathrm{E} 35}$ & 1993 & CABG & 43 & CB: 64 & 37 & 5 & $\begin{array}{l}\text { Warm cardioplegia was } \\
\text { associated with comparable } \\
\text { morbidity and mortality } \\
\text { compared with cold } \\
\text { cardioplegia. }\end{array}$ & $5 / 8$ \\
\hline Yang et $\mathrm{al}^{\mathrm{E} 36}$ & 1994 & Valve & 10 & CC: 10 & 37 & 4 & $\begin{array}{l}\text { No significant differences were } \\
\text { found in clinical outcomes } \\
\text { between warm and cold } \\
\text { cardioplegia. }\end{array}$ & $2 / 8$ \\
\hline Zeriouh et $\mathrm{al}^{\mathrm{E} 47}$ & 2015 & CABG & 506 & CB: 176 & 37 & $4-6$ & $\begin{array}{l}\text { Intermittent warm cardioplegia } \\
\text { was associated with } \\
\text { comparable long-term } \\
\text { outcomes as compared with } \\
\text { intermittent cold cardioplegia. }\end{array}$ & $9 / 9$ \\
\hline
\end{tabular}

MJS, Modified Jadad scale; NOS, Newcastle-Ottawa Quality Assessment Scale; $C A B G$, coronary artery bypass graft; $C B$, cold blood; $N R$, not reported; $C C$, cold crystalloid; $W$, warm; $L$, lukewarm; $L C O S$, low cardiac output syndrome. 
were identified; after full-text screening of 43 papers, only 16 papers published after 2009 met the inclusion criteria. Together with the papers included by Fan and colleagues, ${ }^{3}$ there were 35 RCTs and 11 observational studies (Table 1). A total of 15,428 patients were included (7780 in warm cardioplegia arm, 7648 in cold cardioplegia arm). Electronic search of ClinicalTrials.gov revealed an ongoing RCT (NCT04203680) comparing cold histidine-tryptophan-ketoglutarate solution versus warm blood cardioplegia in CABG, with 30-day mortality as the primary outcome.

Critical appraisal of the included studies was performed using the modified Jadad scale or NOS, as summarized in Table E1 and Table E2, respectively. Overall, 18 of 35 RCTs scored 5 points or greater in modified Jadad scale, with all observational studies scoring 7 points or greater in NOS, and were classified as low risk of bias. Significant proportion of studies included before 2009 were classified as having high risk of bias, mainly due to inappropriate randomization methods and nonblinded studies.

Critical appraisal of meta-analysis by Fan and colleagues in $2010^{3}$ was performed using the AMSTAR 2 tool, $^{6}$ as summarized in Online data supplement. It showed that the systemic review was of moderate quality.

Baseline characteristics of included patients in studies after previous meta-analysis were summarized in Table E3. Other related baseline characteristics (smoker, European System for Cardiac Operative Risk Evaluation, European System for Cardiac Operative Risk Evaluation II, dyslipidemia, peripheral vascular disease, chronic kidney disease, previous AF, chronic obstructive pulmonary disease, previous stroke, previous MI) were not reported, as they were included by fewer than 10 studies.

A pairing table (Table E4) was constructed to indicate outcomes reported by individual studies. All primary outcomes were supported by at least 15 studies (mortality 31 , MI 32, LCOS 15, IABP use 20, stroke 17, new AF 17), whereas secondary outcomes were supported at least 7 studies (AKI 7, hospital LOS 9, ICU LOS 10).

There were no statistically significant differences in all outcomes (mortality, MI, LCOS, IABP use, stroke, new AF, AKI, hospital LOS, and ICU LOS) between warm and cold cardioplegia, with results summarized in Table 2 . Forest plots of outcomes reported by most studies (ie, mortality and MI) were shown in Figures 2 and 3, respectively. None of the primary outcomes exhibited significant heterogeneity. Only hospital LOS and ICU LOS had significant heterogeneity.

A prespecified subgroup analysis was performed on primary outcomes for studies published since Fan and colleagues in 2010, ${ }^{3}$ with results summarized in Table E5 and forest plots included in Figures 2 and 3 and Figures E1-E4. All of the primary outcomes remained statistically insignificant without significant heterogeneity.

Subgroup analysis were also performed on (1) randomized controlled studies, (2) studies of low risk of bias, (3) CABG surgeries, and (4) studies with cold blood versus those with cold crystalloid cardioplegia, with results summarized in Table 3, Table E6, Table E7, and Table E8, respectively. Most outcomes remained statistically insignificant, with heterogeneity qualitatively unchanged. Exceptions included hospital LOS (WMD $-0.84[-1.59$, $-0.10], P=.03$ ) in studies of low risk of bias; and IABP use (RR 0.65 [0.43-0.99], $P=.04$ ) in warm blood versus cold crystalloid cardioplegia, both favoring warm cardioplegia.

Publication bias was assessed visually by funnel plots for outcomes with at least 10 studies (mortality, MI, LCOS, IABP use, stroke, new AF, and ICU LOS) (Figures E5-

TABLE 2. Summary of primary and secondary outcomes

\begin{tabular}{lccc}
\hline & RR or WMD [95\% CI] & P value & Heterogeneity \\
\hline Mortality & RR $0.99[0.80-1.24]$ & .96 & $\mathrm{I}^{2}=0 \%, \chi^{2}=15.47, P=.98$ \\
MI & RR $0.93[0.78-1.12]$ & .48 & $\mathrm{I}^{2}=0 \%, \chi^{2}=18.13, P=.96$ \\
LCOS & RR $0.98[0.64-1.50]$ & .92 & $\mathrm{I}^{2}=36 \%, \chi^{2}=21.91$, \\
& & $P=.08$ & $\mathrm{I}^{2}=0 \%, \chi^{2}=12.70, P=.69$ \\
IABP use & RR $0.95[0.70-1.28]$ & .72 & $\mathrm{I}^{2}=0 \%, \chi^{2}=10.00, P=.76$ \\
Stroke & RR $1.19[0.83-1.69]$ & .35 & $\mathrm{I}^{2}=19 \%, \chi^{2}=19.79$, \\
New AF & RR $1.08[0.92-1.26]$ & .34 & $P=.23$ \\
AKI & RR $0.94[0.59-1.48]$ & .78 & $\mathrm{I}^{2}=0 \%, \chi^{2}=5.50, P=.48$ \\
Hospital LOS & WMD $-0.60[-1.40,0.20]$ & .14 & $\mathrm{I}^{2}=69 \%, \chi^{2}=22.69$, \\
& & $P=.002$ & $\mathrm{I}^{2}=88 \%, \chi^{2}=76.45$, \\
ICU LOS & WMD $-0.12[-0.56,0.32]$ & .60 & $P<.00001$
\end{tabular}

$R R$, Relative risk; $W M D$, weighted mean difference; $C I$, confidence interval; $M I$, myocardial infarction; $L C O S$, low cardiac output syndrome; $I A B P$, intra-aortic balloon pump; $A F$, atrial fibrillation; $A K I$, acute kidney injury; $L O S$, length of stay; $I C U$, intensive care unit. 


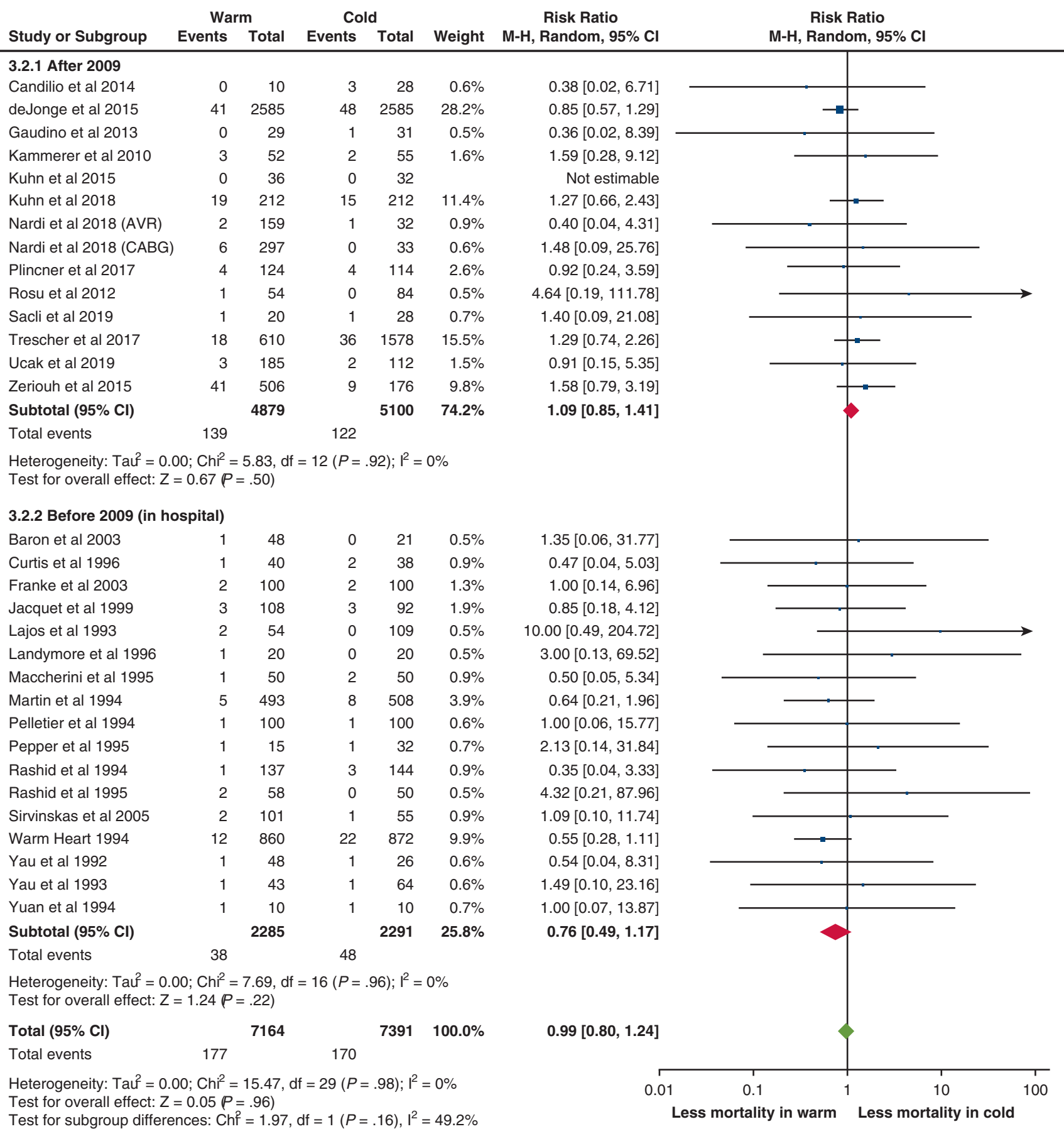

FIGURE 2. Forest plot for mortality. $M-H$, Mantel-Haenszel; $C I$, confidence interval.

E11). No asymmetries were detected, indicating low risk of publication bias.

Sensitivity analysis was performed for all the outcomes using the leave-one-out method. Removal of individual studies from the analysis did not alter the statistical significance, except for the exclusion of Nardi and colleagues ${ }^{9}$ in hospital LOS, which would result in statistically significant $(P=.04)$ shorter LOS in warm cardioplegia arm.
TSA was performed for all the outcomes without significant heterogeneity. The $\mathrm{Z}$ value is the test statistic and $|\mathrm{Z}|=1.96$ corresponds to a $P=.05$, with greater $\mathrm{Z}$ values corresponding to lower $P$ values. The $\mathrm{Z}$-score curve for mortality (adjusted RR 1.0 [0.77-1.31], $P=.98$; $\mathrm{I}^{2}=0 \%$; Figure 4, A), MI (adjusted RR 0.91 [0.74-1.11], $P=.35 ; \mathrm{I}^{2}=0 \%$; Figure $4, B$ ), LCOS (adjusted RR, 1.19 [0.59-2.40], $P=.61 ; \mathrm{I}^{2}=46 \%$; Figure 4,C), and 


\begin{tabular}{|c|c|c|c|c|c|c|c|c|c|}
\hline Study or Subgroup & \multicolumn{2}{|c|}{ Warm } & \multicolumn{2}{|c|}{ Cold } & Weight & $\begin{array}{c}\text { Risk Ratio } \\
\text { M-H, Random, } 95 \% \text { Cl }\end{array}$ & \multicolumn{2}{|c|}{$\begin{array}{l}\text { Risk Ratio } \\
\text { M-H, Random, } 95 \% \text { Cl }\end{array}$} & \\
\hline \multicolumn{3}{|l|}{ 3.1.1 After 2009} & & & & & & & \\
\hline Baig et al 2015 & 9 & 94 & 21 & 121 & $6.3 \%$ & $0.55[0.27,1.15]$ & & & \\
\hline Candilio et al 2014 & 0 & 10 & 1 & 28 & $0.3 \%$ & $0.88[0.04,19.99]$ & & & \\
\hline Mourad et al 2016 & 0 & 50 & 0 & 50 & & Not estimable & & & \\
\hline Nardi et al 2018 (AVR) & 6 & 159 & 0 & 32 & $0.4 \%$ & $2.68[0.15,46.44]$ & & & \\
\hline Nardi et al 2018 (CABG) & 13 & 297 & 1 & 33 & $0.9 \%$ & $1.44[0.20,10.69]$ & & & \\
\hline Plincner et al 2017 & 11 & 124 & 9 & 114 & $4.8 \%$ & $1.12[0.48,2.61]$ & - & & \\
\hline Rosu et al 2012 & 3 & 54 & 1 & 84 & $0.7 \%$ & $4.67[0.50,43.71]$ & & & \\
\hline Trescher et al 2017 & 10 & 610 & 11 & 1578 & $4.7 \%$ & $2.35[1.00,5.51]$ & & & \\
\hline Ucak et al 2019 & 1 & 185 & 0 & 112 & $0.3 \%$ & $1.82[0.07,44.36]$ & & & \\
\hline Zeriouh et al 2015 & 59 & 506 & 25 & 176 & $18.0 \%$ & $0.82[0.53,1.27]$ & $\rightarrow$ & & \\
\hline Subtotal $(95 \% \mathrm{Cl})$ & & 2089 & & 2328 & $36.5 \%$ & $1.05[0.71,1.57]$ & & & \\
\hline Total events & 112 & & 69 & & & & & & \\
\hline \multicolumn{10}{|c|}{$\begin{array}{l}\text { Heterogeneity: } \text { Tau }^{2}=0.06 ; \mathrm{Chi}^{2}=9.82, \mathrm{df}=8(P=.28) ; \mathrm{I}^{2}=19 \% \\
\text { Test for overall effect: } Z=0.26(P=.80)\end{array}$} \\
\hline \multicolumn{10}{|l|}{ 3.1.2 Before 2009} \\
\hline Ali et al 1994 & 2 & 38 & 2 & 38 & $0.9 \%$ & $1.00[0.15,6.74]$ & & & \\
\hline Ascione et al 2002 & 1 & 19 & 0 & 16 & $0.3 \%$ & $2.55[0.11,58.60]$ & & & \\
\hline Chocron et al 2000 & 0 & 45 & 1 & 45 & $0.3 \%$ & $0.33[0.01,7.97]$ & & & \\
\hline Curtis et al 1996 & 2 & 40 & 0 & 38 & $0.4 \%$ & $4.76[0.24,95.96]$ & & & \\
\hline Elwatidy et al 1999 & 1 & 47 & 1 & 81 & $0.5 \%$ & $1.72[0.11,26.92]$ & & & \\
\hline Engelman et al 1996 & 1 & 93 & 0 & 37 & $0.3 \%$ & $1.21[0.05,29.12]$ & & & \\
\hline Franke et al 2003 & 2 & 100 & 3 & 100 & $1.1 \%$ & $0.67[0.11,3.90]$ & & & \\
\hline Hayashida et al 1994 & 1 & 48 & 0 & 24 & $0.3 \%$ & $1.53[0.06,36.23]$ & & & \\
\hline Hayashida et al 1995 & 0 & 28 & 1 & 14 & $0.3 \%$ & $0.17[0.01,3.98]$ & $\leftarrow$ & & \\
\hline Honkonen et al 1997 & 0 & 15 & 2 & 14 & $0.4 \%$ & $0.19[0.01,3.60]$ & $\leftarrow$ & & \\
\hline Isomura et al 1995 & 1 & 29 & 0 & 26 & $0.3 \%$ & $2.70[0.11,63.52]$ & & & \\
\hline Jacquet et al 1999 & 5 & 108 & 5 & 92 & $2.3 \%$ & $0.85[0.25,2.85]$ & & & \\
\hline Lajos et al 1993 & 1 & 54 & 3 & 109 & $0.7 \%$ & $0.67[0.07,6.32]$ & & & \\
\hline Landymore et al 1996 & 1 & 20 & 1 & 20 & $0.5 \%$ & $1.00[0.07,14.90]$ & & & \\
\hline Martin et al 1994 & 6 & 493 & 4 & 508 & $2.2 \%$ & $1.55[0.44,5.44]$ & & & \\
\hline Pelletier et al 1994 & 2 & 100 & 4 & 100 & $1.2 \%$ & $0.50[0.09,2.67]$ & & & \\
\hline Rashid et al 1994 & 8 & 137 & 7 & 144 & $3.5 \%$ & $1.20[0.45,3.22]$ & & & \\
\hline Rashid et al 1995 & 3 & 58 & 2 & 50 & $1.1 \%$ & $1.29[0.22,7.43]$ & & & \\
\hline Sirvinskas et al 2005 & 2 & 101 & 2 & 55 & $0.9 \%$ & $0.54[0.08,3.76]$ & & & \\
\hline Warm Heart 1994 & 85 & 843 & 93 & 842 & $44.1 \%$ & $0.91[0.69,1.21]$ & & & \\
\hline Yau et al 1992 & 2 & 48 & 3 & 26 & $1.1 \%$ & $0.36[0.06,2.03]$ & & - & \\
\hline Yau et al 1993 & 1 & 43 & 2 & 64 & $0.6 \%$ & $0.74[0.07,7.95]$ & & & \\
\hline Subtotal $(95 \% \mathrm{Cl})$ & & 2507 & & 2443 & $63.5 \%$ & $0.91[0.72,1.15]$ & 4 & & \\
\hline Total events & 127 & & 136 & & & & & & \\
\hline \multicolumn{10}{|c|}{$\begin{array}{l}\text { Heterogeneity: } \mathrm{Tau}^{2}=0.00 ; \mathrm{Chi}^{2}=8.19, \mathrm{df}=21(P=.99) ; \mathrm{I}^{2}=0 \% \\
\text { Test for overall effect: } Z=0.79(P=.43)\end{array}$} \\
\hline Total $(95 \% \mathrm{Cl})$ & & 4596 & & 4771 & $100.0 \%$ & $0.93[0.78,1.12]$ & & & \\
\hline Total events & 239 & & 205 & & & & & & \\
\hline \multicolumn{6}{|c|}{$\begin{array}{l}\text { Heterogeneity: } \mathrm{Tau}^{2}=0.00 ; \mathrm{Chi}^{2}=18.13, \mathrm{df}=30(P=.96) ; \mathrm{I}^{2}=0 \% \\
\text { Test for overall effect: } Z=0.71(P=.48) \\
\text { Test for subgroup differences: } \mathrm{Chi}^{2}=0.38, \mathrm{df}=1(P=.54), \mathrm{I}^{2}=0 \%\end{array}$} & 0.01 & 0.1 & & 10 \\
\hline
\end{tabular}

FIGURE 3. Forest plot for MI. $M-H$, Mantel-Haenszel; $C I$, confidence interval; $M I$, myocardial infarction.

AF (adjusted RR, 1.07 [0.86-1.33], $P=.49 ; \mathrm{I}^{2}=28 \%$; Figure $4, D$ ) crossed the required information size boundary, indicating current evidence was sufficient in concluding that there were no significant differences between both arms. The Z-score curve for IABP use (adjusted RR, 0.99 [0.60-1.64], $P=.96 ; \mathrm{I}^{2}=20 \%$;
Figure 5, A), stroke (adjusted RR, 1.03 [0.57-1.87], $P=.89 ; \mathrm{I}^{2}=0 \%$; Figure $5, B$ ), and AKI (adjusted RR, 0.97 [0.51-1.84], $P=.92 ; \mathrm{I}^{2}=23 \%$; Figure $5, C$ ) crossed the futility boundary, indicating current evidence was sufficient in ruling out a $20 \%$ RR reduction in these outcomes. 
TABLE 3. Summary of primary and secondary outcomes in randomised controlled studies

\begin{tabular}{llcc}
\hline & RR or WMD [95\% CI] & $\boldsymbol{P}$ value & Heterogeneity \\
\hline Mortality & RR $0.80[0.54-1.19]$ & .27 & $\mathrm{I}^{2}=0 \%, \chi^{2}=8.76, P=.99$ \\
MI & RR $0.91[0.73-1.15]$ & .45 & $\mathrm{I}^{2}=0 \%, \chi^{2}=8.37, P=1.00$ \\
LCOS & RR $0.85[0.57-1.27]$ & .44 & $\mathrm{I}^{2}=24 \%, \chi^{2}=14.43, P=.21$ \\
IABP use & RR $1.19[0.82-1.74]$ & .37 & $\mathrm{I}^{2}=0 \%, \chi^{2}=7.06, P=.93$ \\
Stroke & RR $1.43[0.91-2.24]$ & .12 & $\mathrm{I}^{2}=0 \%, \chi^{2}=6.85, P=.74$ \\
New AF & RR $1.06[0.87-1.28]$ & .56 & $\mathrm{I}^{2}=0 \%, \chi^{2}=8.99, P=.53$ \\
AKI & RR $0.85[0.20-3.54]$ & .82 & $\mathrm{I}^{2}=0 \%, \chi^{2}=0.28, P=.60$ \\
Hospital LOS & WMD $-0.44[-1.54,0.67]$ & .44 & $\mathrm{I}^{2}=47 \%, \chi^{2}=3.80, P=.15$ \\
ICU LOS & WMD $0.24[-0.34,0.83]$ & .42 & $\mathrm{I}^{2}=79 \%, \chi^{2}=13.99, P=.003$ \\
\hline$R R$, R
\end{tabular}

$R R$, Relative risk; $W M D$, weighted mean difference; $C I$, confidence interval; $M I$, myocardial infarction; $L C O S$, low cardiac output syndrome; $I A B P$, intra-aortic balloon pump; $A F$, atrial fibrillation; $A K I$, acute kidney injury; $L O S$, length of stay; $I C U$, intensive care unit.
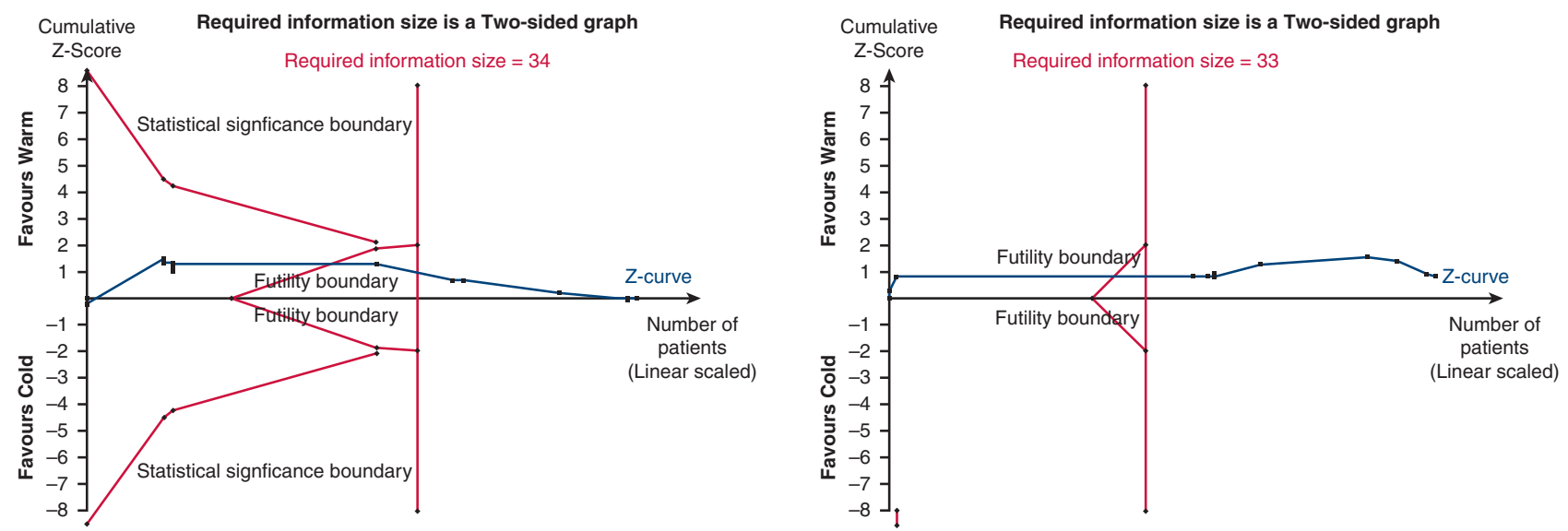

A

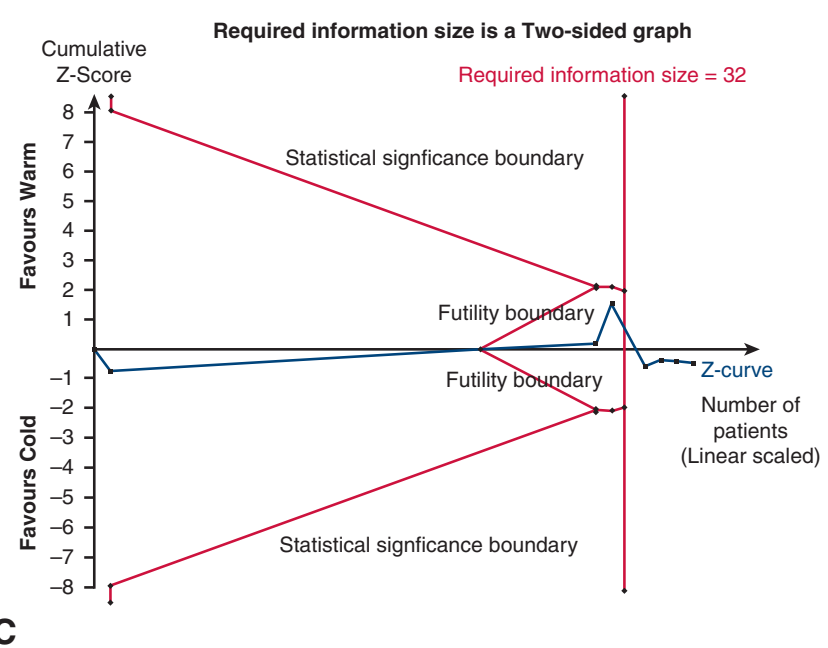

B

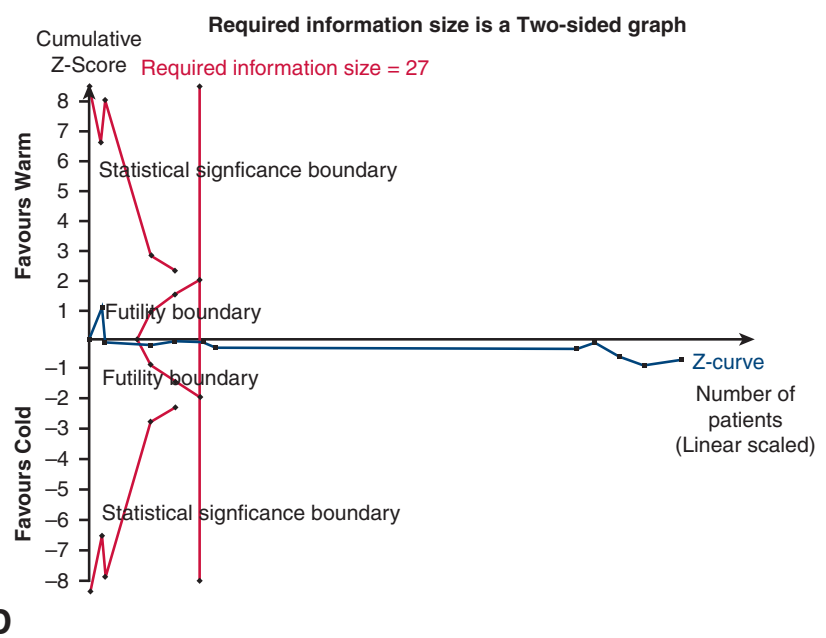

FIGURE 4. Trial sequential analysis of (A) mortality, (B) myocardial infarction, (C) low cardiac output syndrome, and (D) atrial fibrillation. $Z$ value is the test statistic and $|Z|=1.96$ corresponds to a $P=.05$. The required information size to detect or reject the $20 \%$ relative risk reduction found in random-effects model meta-analysis is calculated using diversity found in meta-analysis, with double-sided $\alpha=0.05$ and $\beta=0.20$ (power of $80 \%$ ). 

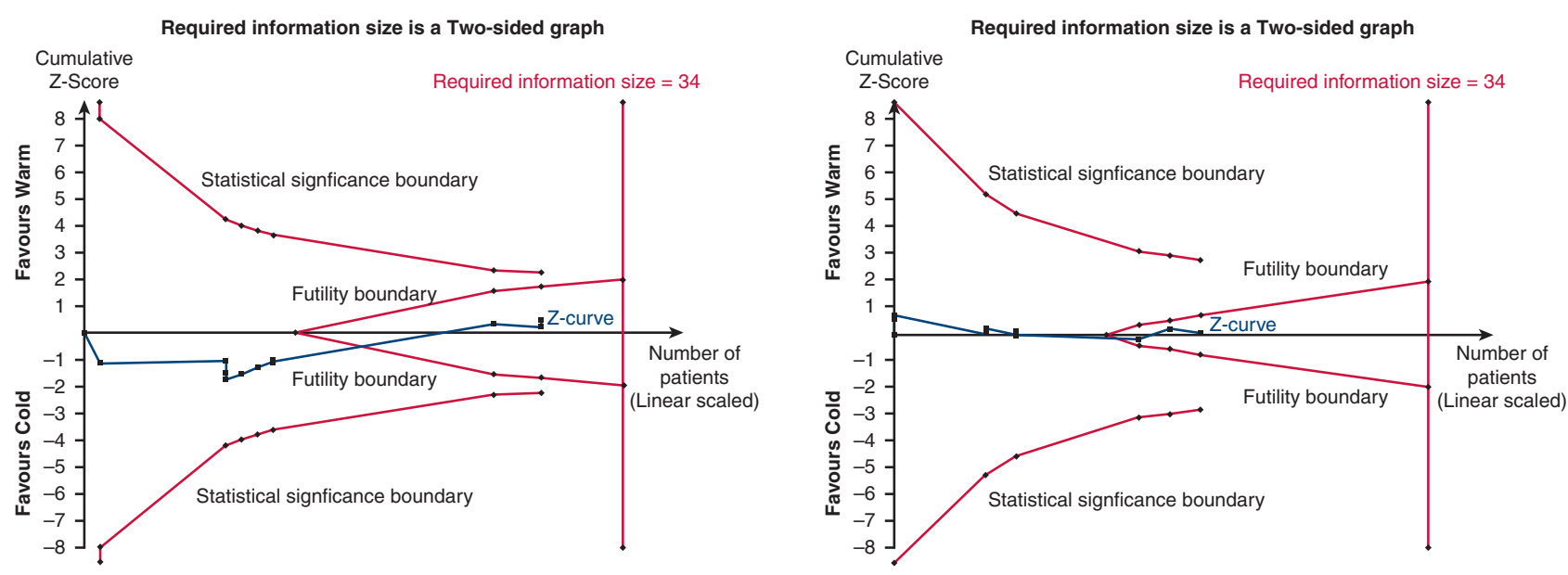

A

B

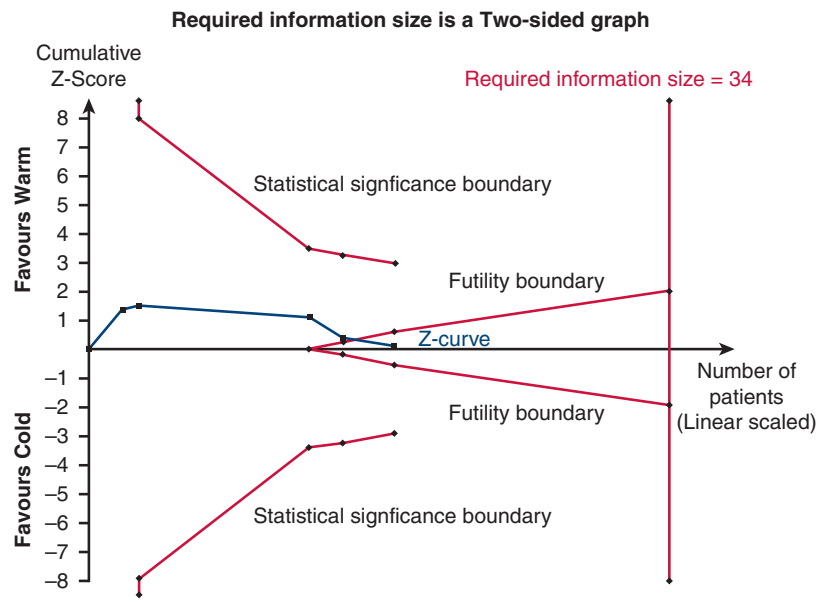

C

FIGURE 5. Trial sequential analysis of (A) intra-aortic balloon pump use, (B) stroke, and (C) acute kidney injury. $Z$ value is the test statistic and $|Z|=1.96$ corresponds to a $P=.05$. The required information size to detect or reject the $20 \%$ relative risk reduction found in random-effects model meta-analysis is calculated using diversity found in meta-analysis, with double-sided $\alpha=0.05$ and $\beta=0.20$ (power of $80 \%$ ).

\section{DISCUSSION}

In this study, we compared operative and clinical outcomes of warm and cold cardioplegia. No significant differences were found between both arms for all outcomes. TSA showed that current evidence was conclusive to rule out $20 \% \mathrm{RR}$ reduction in the following outcomes: mortality, MI, LCOS, IABP use, stroke, new AF, and AKI (Figure 6).

Overall, our results confirmed the findings by Fan and colleagues $^{3}$ that warm and cold cardioplegia were not significantly different in efficacy and safety, further providing a broader look at clinical and operative outcomes. Despite not exhibiting statistically significant subgroup differences, diverging trends were found upon subgroup analysis, suggestive of subtle differences between the subgroups. However, when analyzing only studies with low risk of bias, these numerical trends disappeared. This suggests that such trends might have been the result of bias, possibly due to unclear or inappropriate randomization methods and a lack of blinding in some trials. Furthermore, when we compared cold blood with cold crystalloid cardioplegia, the outcomes of mortality, LCOS, IABP use, stroke, and AKI showed trends in opposite directions, with subgroup differences of $P=.27, P=.21, P=.009$, $P=.09$, and $P=.08$, respectively. The 2014 metaanalysis by Zeng and colleagues ${ }^{10}$ suggested that subtle subgroup differences can lead to drastically different outcomes. In their study, there were significantly less postoperative $\mathrm{MI}$ in cold blood cardioplegia; however, there were no significant differences in mortality, $\mathrm{AF}$, and stroke between cold blood versus crystalloid cardioplegia. This may have been the reason for the statistically insignificant trends in our subgroup analysis, as current studies may not have been designed to specifically compare cold blood versus cold crystalloid cardioplegia. Nonetheless, the results by 
Warm versus cold cardioplegia in cardiac surgery: A meta-analysis with trial sequential analysis

35 randomized controlled trials +11 observational studies

Total:15,428 patients ( 7780 warm cardioplegia vs 7648 cold cardioplegia)

Risk Ratio

M-H, Random, 95\% Cl
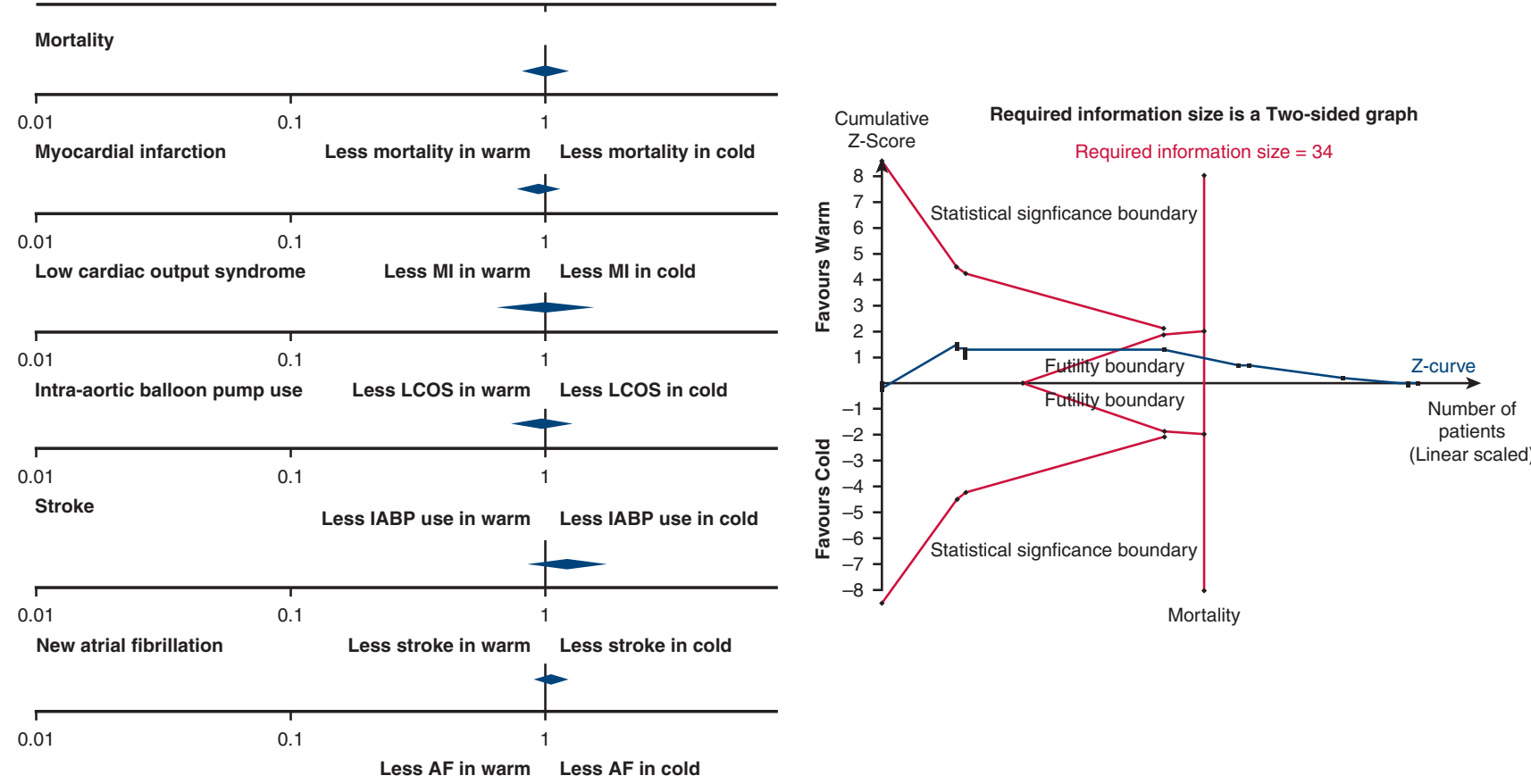

The choice of warm versus cold cardioplegia solution remains the surgeon's preference.

Abbreviations:

AF, atrial fibrillation. $\mathrm{Cl}$, confidence interval. M-H, Mantel-Haenszel. IABP, intra-aortic balloon pump.

LCOS, low cardiac output syndrome. MI, myocardial infarction.

FIGURE 6. Warm versus cold cardioplegia in cardiac surgery: a meta-analysis with trial sequential analysis. Forty-six studies, with 15,428 patients were included in analysis ( 35 randomized controlled trials +11 observational studies). No significant differences were found between two arms in post-operative mortality, myocardial infarction, low cardiac output syndrome, intra-aortic balloon pump use, stroke, and new atrial fibrillation as shown in the Forest plots. Trial sequential analysis of mortality was shown signifying current evidences were conclusive. In conclusion, choice of warm versus cold cardioplegia remains surgeon's preference.

Zeng and colleagues ${ }^{10}$ were limited by high risks of bias and other possible confounders; therefore, more studies are needed to evaluate the effects of blood versus crystalloid cardioplegia.

All primary outcomes and AKI exhibited insignificant heterogeneity, whereas TSA showed conclusive results. While all primary outcomes were supported by at least 15 studies, suggesting uniformity of the included studies regarding the outcome of interest. In contrast, hospital LOS and ICU LOS displayed significant heterogeneity. This could be contributed by several factors, including differences in local practices, the admission and discharge criteria of ICU, experience of surgeons' etcetera. Heterogeneity remained high despite stratification by subgroups, suggesting that variability was less likely to be caused by differences in publication year, biased studies, type of surgery, or composition of cardioplegia solution. Although such significant heterogeneity limited the strength of our findings, our analysis represented the most up-to-date evidence. However, there is a need for further studies delineating factors affecting the aforementioned outcomes, along with trials controlling for the aforementioned factors.

A survey performed by Ali and colleagues ${ }^{11}$ in 2018 revealed significant variation in the international practice of myocardial protection, with no clear consensus on the use of cardioplegia currently. Variability exists in composition and delivery method of cardioplegic solutions. However, limited by evidences available, subgroup analysis was not performed in those aspects mentioned. Most of our included studies administered cardioplegic solutions in antegrade fashion. Composition of blood and crystalloid solutions varies among studies, including mixture of blood with other solutions, Custodiol solution, Buckberg solution, St Thomas Hospital solution, and Del Nido solution, etc. Further studies should be done focusing on factors that were not evaluated in this meta-analysis. 
The safety and efficacy of different types of crystalloid solutions (eg, histidine-tryptophan-ketoglutarate solution, St Thomas solution) warrants further investigations, as exemplified in the study by Pizano and colleagues. ${ }^{12}$ Histidine-tryptophan-ketoglutarate solution is a widely used cardioplegic and organ-preserving solution; however, despite its widespread use, it is seldom studied in comparison with blood cardioplegia. Del Nido solution was initially intended for pediatric surgeries and is now extended to adult cardiac surgeries. Ler and colleagues, ${ }^{13}$ in a meta-analysis performed in 2020, compared Del Nido versus St Thomas cardioplegic solution, showing similar postoperative outcomes.

Besides composition of cardioplegic solution, mode of administration should be further explored. Gambardella and colleagues $^{14}$ performed a meta-analysis in 2019 comparing single versus multidose cardioplegia, suggesting that more studies were needed to compare effects of different solution types, as current evidence were not yet conclusive. In addition, terminal hot-shot cardioplegia was proposed as a potential way to improve clinical outcomes, yet a systematic review performed by Volpi and colleagues $^{15}$ in 2019 concluded that there was insufficient evidence to evaluate its clinical merits. Mallidi and colleagues ${ }^{16}$ conducted an observational study, suggesting that warm blood cardioplegia was associated with better long-term survival and less late MI than cold blood cardioplegia. However, there are few studies in the current literature that investigate the long-term effects of warm versus cold cardioplegia, which in fact are important concerns that should be further explored.

In fact, the choice of cardioplegic solutions might be based on other perceived benefits that were not investigated in this meta-analysis. At our unit, which consisted of 22 consultant cardiac and aortic surgeons, choice of cardioplegic temperature remained surgeons' preferences. The majority of our team members preferred cold cardioplegia, aiming for metabolic inhibition and thus less energy consumption. In the meta-analysis by Ler and colleagues, ${ }^{13}$ the rate of defibrillation, aortic crossclamp time, and cardiopulmonary bypass time were listed as primary outcomes. This might suggest future directions of investigating for other outcomes.

This meta-analysis has a few limitations. First, one major confounder was the variation in means of administrating the cardioplegia solutions: intermittent versus continuous and antegrade versus retrograde. This meta-analysis was not designed to investigate these factors; thus, it was unclear the impact of these factors on our findings. Second, a significant proportion of the studies included that were published before 2009 had a high risk of bias; however, we attempted to mitigate this issue by performing a subgroup analysis of studies with low risk of bias. Third, the results of observational studies published after 2009 were pooled with RCTs, which may add on to confounders and selection bias. To address this issue, we performed a subgroup analysis for RCTs, which showed no changes of statistical significances of all outcomes. In addition to that, the observational studies included in this meta-analysis were of low risk of bias, and their value lies in their representation of real-world experience. Fourth, this study based on and updated the findings of previously meta-analysis by Fan and colleagues in 2010. ${ }^{3}$ The results might therefore be inaccurate if it was of poor quality. Thus, a quality assessment was performed, showing that it was of moderate quality without major methodologic flaws. Fifth, the studies included in this meta-analysis spanned over 27 years, during which time surgical techniques and clinical practices have advanced significantly. To mitigate this issue, we performed a subgroup analysis of studies published after 2009. Nonetheless, this cutoff of publication time was merely based on the publication of the last meta-analysis on this topic, and the time of publication remains a potential confounder. Finally, only studies in English language were included; therefore, it is possible that relevant nonEnglish studies were omitted.

In conclusion, this systematic review and meta-analysis concluded that there were no significant differences in postoperative rates of mortality, MI, LCOS, IABP use, stroke, new AF, and AKI, between the use of warm and cold cardioplegia. The choice of warm versus cold cardioplegia solution remains the surgeon's preference. Nonetheless, further studies should evaluate any differences between various compositions and modes of administrating cardioplegic solutions, with greater exploration on the long-term effects of warm versus cold cardioplegia.

\section{Conflict of Interest Statement}

The authors reported no conflicts of interest. The funding was provided by University College London Open Access Team, Main Library; University College London, UK.

The Journal policy requires editors and reviewers to disclose conflicts of interest and to decline handling or reviewing manuscripts for which they may have a conflict of interest. The editors and reviewers of this article have no conflicts of interest.

We hereby thank the following individuals for their assistance and effort in this project: Shaik Ashraf Bin Shaik Ismail (University of Liverpool Medical School) for title and abstract screening, full-text screening, data extraction; Wing Yan Elizabeth Wong (Brighton and Sussex Medical School, University of Sussex) for data extraction; and Chun Ming Chiu (Brighton and Sussex Medical School, University of Sussex) for data extraction.

\section{References}

1. Mauney MC, Kron IL. The physiologic basis of warm cardioplegia. Ann Thorac Surg. 1995;60:819-23.

2. The Warm Heart Investigators. Randomised trial of normothermic versus hypothermic coronary bypass surgery. Lancet. 1994;343:559-63. 
3. Fan Y, Zhang A-M, Xiao Y-B, Weng Y-G, Hetzer R. Warm versus cold cardioplegia for heart surgery: a meta-analysis. Eur J Cardiothorac Surg. 2010;37:912-9.

4. Liberati A, Altman DG, Tetzlaff J, Mulrow C, Gøtzsche PC, Ioannidis JPA, et al The PRISMA statement for reporting systematic reviews and meta-analyses of studies that evaluate healthcare interventions: explanation and elaboration. BMJ. 2009;339:b2700.

5. Higgins J, Thomas J, Chandler J, Cumpston M, Li T, Page MJ, et al., eds. Cochrane Handbook for Systematic Reviews of Interventions version 6.0. 2019. Cochrane. Available at: www.training.cochrane.org/handbook. Accessed July 30, 2020.

6. Shea BJ, Reeves BC, Wells G, Thuku M, Hamel C, Moran J, et al. AMSTAR 2: a critical appraisal tool for systematic reviews that include randomised or non-randomised studies of healthcare interventions, or both. BMJ. 2017;358: $1-9$.

7. Wetterslev J, Jakobsen JC, Gluud C. Trial sequential analysis in systematic reviews with meta-analysis. BMC Med Res Methodol. 2017;17: 39.

8. Chan JSK, Harky A. Trial sequential analysis in meta-analyses: a clinically oriented approach with real world example. J Thorac Cardiovasc Surg. June 27, 2020 [Epub ahead of print].

9. Nardi P, Vacirca SR, Russo M, Colella DF, Bassano C, Scafuri A, et al. Cold crystalloid versus warm blood cardioplegia in patients undergoing aortic valve replacement. J Thorac Dis. 2018;10:1490-9.

10. Zeng J, He W, Qu Z, Tang Y, Zhou Q, Zhang B. Cold blood versus crystalloid cardioplegia for myocardial protection in adult cardiac surgery: a meta- analysis of randomized controlled studies. J Cardiothorac Vasc Anesth. 2014; 28:674-81.

11. Ali JM, Miles LF, Abu-Omar Y, Galhardo C, Falter F. Global cardioplegia practices: results from the global cardiopulmonary bypass survey. J Extra Corpor Technol. 2018;50:83-93.

12. Pizano A, Montes FR, Carreño M, Echeverri D, Umaña JP. Histidine-tryptophanketoglutarate solution versus blood cardioplegia in cardiac surgery: a propensityscore matched analysis. Heart Surg Forum. 2018;21:E158-64.

13. Ler A, Sazzad F, Ong GS, Kofidis T. Comparison of outcomes of the use of Del Nido and St. Thomas cardioplegia in adult and paediatric cardiac surgery: a systematic review and meta-analysis. Perfusion. 2020;35:724-35.

14. Gambardella I, Gaudino MFL, Antoniou GA, Rahouma M, Worku B, Tranbaugh RF, et al. Single- versus multidose cardioplegia in adult cardiac surgery patients: a meta-analysis. J Thorac Cardiovasc Surg. 2020;160: 1195-202.e2.

15. Volpi S, Ali JM, De Silva R. Does the use of a hot-shot lead to improved outcomes following adult cardiac surgery? Interact Cardiovasc Thorac Surg. 2019;28:473-7.

16. Mallidi HR, Sever J, Tamariz M, Singh S, Hanayama N, Christakis GT, et al. The short-term and long-term effects of warm or tepid cardioplegia. J Thorac Cardiovasc Surg. 2003;125:711-20.

Key Words: cardiac surgeries, cold cardioplegia, warm cardioplegia, meta-analysis, trial sequential analysis 


\section{E-References}

E1. Fan Y, Zhang A-M, Xiao Y-B, Weng Y-G, Hetzer R. Warm versus cold cardioplegia for heart surgery: a meta-analysis. Eur J Cardiothorac Surg. 2010;37: 912-9.

E2. Ali IM, Kinley CE. The safety of intermittent warm blood cardioplegia. Eur J Cardiothorac Surg. 1994;8:554-6.

E3. Ascione R, Caputo M, Gomes WJ, Lotto AA, Bryan AJ, Angelini GD, et al. Myocardial injury in hypertrophic hearts of patients undergoing aortic valve surgery using cold or warm blood cardioplegia. Eur J Cardiothorac Surg. 2002:21:440-6.

E4. Baron O, Roussel JC, Delaroche O, Péron S, Duveau D. Prospective clinical and biological comparison of three blood cardioplegia techniques in low-risk CABG patients: better is worse than good enough. Vascular. 2003;11:489-95.

E5. Chello M, Mastroroberto P, Romano R, Ascione R, Pantaleo D, De Amicis V. Complement and neutrophil activation during cardiopulmonary bypass: a randomized comparison of hypothermic and normothermic circulation. Eur J Cardiothorac Surg. 1997;11:162-8.

E6. Chello M, Mastroroberto P, Patti G, D’Ambrosio A, Di Sciascio G, Covino E. Intermittent warm blood cardioplegia induces the expression of heat shock protein-72 by ischemic myocardial preconditioning. Vascular. 2003;11: 367-74

E7. Chocron S, Kaili D, Yan Y, Toubin G, Latini L, Clement F, et al. Intermediate lukewarm $\left(20^{\circ} \mathrm{C}\right)$ antegrade intermittent blood cardioplegia compared with cold and warm blood cardioplegia. J Thorac Cardiovasc Surg. 2000;119:610-6.

E8. Curtis JJ, Nawarawong W, Walls JT, Schmaltz RA. Continuous warm blood cardioplegia: a randomized prospective clinical comparison. Int J Angiol. 1996;5: 212-8.

E9. Dar MI. Cold crystalloid versus warm blood cardioplegia for coronary artery bypass surgery. Ann Thorac Cardiovasc Surg. 2005;11:382-5.

E10. Elwatidy AMF, Fadalah MA, Bukhari EA, Aljubair KA, Syed A, Ashmeg AK, et al. Antegrade crystalloid cardioplegia vs antegrade/retrograde cold and tepid blood cardioplegia in CABG. Ann Thorac Surg. 1999;68:447-53.

E11. Engelman RM, Pleet AB, Rousou JA, Flack JE, Deaton DW, Gregory CA, et al. What is the best perfusion temperature for coronary revascularization? J Thorac Cardiovasc Surg. 1996;112:1622-33.

E12. Franke UFW, Korsch S, Wittwer T, Albes JM, Wippermann J, Kaluza M, et al. Intermittent antegrade warm myocardial protection compared to intermittent cold blood cardioplegia in elective coronary surgery - do we have to change? Eur J Cardiothorac Surg. 2003;23:341-6.

E13. Gaudino M, Pragliola C, Anselmi A, Pieroni M, Paulis SD, Leone A, et al. Randomized trial of HTK versus warm blood cardioplegia for right ventricular protection in mitral surgery. Scand Cardiovasc J. 2013;47:359-67.

E14. Hayashida N, Ikonomidis JS, Weisel RD, Shirai T, Ivanov J, Carson SM, et al. The optimal cardioplegic temperature. Ann Thorac Surg. 1994;58:961-71.

E15. Hayashida N, Weisel RD, Shirai T, Ikonomidis JS, Ivanov J, Carson SM, et al. Tepid antegrade and retrograde cardioplegia. Ann Thorac Surg. 1995;59:723-9.

E16. Honkonen EL, Kaukinen L, Pehkonen EJ, Kaukinen S. Right ventricle is protected better by warm continuous than by cold intermittent retrograde blood cardioplegia in patients with obstructed right coronary artery. Thorac Cardiovasc Surg. 1997;45:182-9.

E17. Isomura T, Hisatomi K, Sato T, Hayashida N, Ohishi K. Interrupted warm blood cardioplegia for coronary artery bypass grafting. Eur J Cardiothorac Surg. 1995;9:133-8.

E18. Jacquet LM, Noirhomme PH, Van Dyck MJ, El Khoury GA, Matta AJ, Goenen MJ, et al. Randomized trial of intermittent antegrade warm blood versus cold crystalloid cardioplegia. Ann Thorac Surg. 1999;67:471-7.

E19. Kammerer I, Nagib R, Hipp G, Premar M, Hansen M, Franke U. Myocardial protection in minimally invasive mitral valve surgery: comparison of the cold-blood cardioplegia of the bretschneider solution and the warm-blood cardioplegia of the calafiore protocol. Arch Clin Exp Surg. 2012;1:14.

E20. Kuhn EW, Choi YH, Pyun JM, Neef K, Liakopoulos OJ, Stamm C, et al. Endothelial injury associated with cold or warm blood cardioplegia during coronary artery bypass graft surgery. Biomed Res Int. 2015;2015:256905.

E21. Lajos T, Espersen C, Lajos P, Fiedler R, Bergsland J, Joyce L. Comparison of cold versus warm cardioplegia. Crystalloid antegrade or retrograde blood? Circulation. 1993;88:II344-9.

E22. Landymore R, Murphy JT, Hall R, Islam M. Randomized trial comparing intermittent antegrade warm blood cardioplegia with multidose cold blood cardioplegia for coronary artery bypass. Eur J Cardiothorac Surg. 1996;10: 179-84.
E23. Maccherini M, Davoli G, Sani G, Rossi P, Giani S, Lisi G, et al. Warm hear surgery eliminates diaphragmatic paralysis. J Card Surg. 1995;10:257-61.

E24. Martin TD, Craver JM, Gott JP, Weintraub WS, Ramsay J, Mora CT, et al. Prospective, randomized trial of retrograde warm blood cardioplegia: myocardial benefit and neurologic threat. Ann Thorac Surg. 1994;57:298-304.

E25. Pelletier LC, Carrier M, Leclerc Y, Cartier R, Wesolowska E, Solymoss BC. Intermittent antegrade warm versus cold blood cardioplegia: a prospective, randomized study. Ann Thorac Surg. 1994;58:41-9.

E26. Pepper JR, Mumby S, Gutteridge JMC. Blood cardioplegia increases plasma iron overload and thiol levels during cardiopulmonary bypass. Ann Thorac Surg. 1995;60:1735-40.

E27. Rashid A, Fabri BM, Jackson M, Desmond MJ, Grech ED, Battistessa SA, et al. A prospective randomised study of continuous warm versus intermittentcold blood cardioplegia for coronary artery surgery: preliminary report. Eur J Cardiothorac Surg. 1994;8:265-9.

E28. Rashid A, Jackson M, Page RD, Desmond MJ, Fabri BM. Continuous warm versus intermittent cold blood cardioplegia for coronary bypass surgery in patients with left ventricular dysfunction. Eur J Cardiothorac Surg. 1995;9:405-9.

E29. Sacl1 H, Kara I, Diler MS, Percin B, Turan AI, Kırali K. The relationship between the use of cold and isothermic blood cardioplegia solution for myocardial protection during cardiopulmonary bypass and the ischemia-reperfusion injury. Ann Thorac Cardiovasc Surg. 2019;25:296-303.

E30. Şirlak M, Eryilmaz S, Yaziciog̀lu L, Kiziltepe U, Inan MB, Taşöz R, et al. Conduction disturbances in coronary artery bypass surgery. Int J Cardiol. 2003;92: 43-8.

E31. Sirvinskas E, Nasvytis L, Raliene L, Vaskelyte J, Toleikis A, Trumbeckaite S. Myocardial protective effect of warm blood, tepid blood, and cold crystalloid cardioplegia in coronary artery bypass grafting surgery. Croat Med J. 2005; 46:879-88

E32. The Warm Heart Investigators. Randomised trial of normothermic versus hypothermic coronary bypass surgery. Lancet. 1994;343:559-63.

E33. Ucak HA, Uncu H. Comparison of del Nido and intermittent warm blood car dioplegia in coronary artery bypass grafting surgery. Ann Thorac Cardiovasc Surg. 2019;25:39-45.

E34. Yau TM, Weisel RD, Mickle DAG, Komeda K, Ivanov J, Carson S, et al. Alternative techniques of cardioplegia. Circulation. 1992;86(5 Suppl):II377-84.

E35. Yau TM, Ikonomidis JS, Weisel RD, Mickle DA, Hayashida N, Ivanov J, et al. Which techniques of cardioplegia prevent ischemia? Ann Thorac Surg. 1993 56:1020-8.

E36. Yang SM, Zhu LB, Li GS, Liu MH, Dong C, Yu YF, et al. Myocardial protection of cold crystalloid and warm blood cardioplegia. A comparative study. Chin Med J (Engl). 1994;107:515-20

E37. Raza Baig MA, Sher-I-Murtaza M, Iqbal A, Ahmad MZ, Rizvi HMFA, Ahmed N, et al. Clinical outcomes of intermittent antegrade warm versus cold blood cardioplegia. J Pak Med Assoc. 2015;65:593-6.

E38. Candilio L, Malik A, Ariti C, Khan SA, Barnard M, Salvo CD, et al. A retrospective analysis of myocardial preservation techniques during coronary artery bypass graft surgery: are we protecting the heart? J Cardiothorac Surg. 2014;9: $1-11$.

E39. De Jonge M, Van Boxtel AG, Soliman Hamad MA, Mokhles MM, Bramer S Osnabrugge RLJ, et al. Intermittent warm blood versus cold crystalloid cardioplegia for myocardial protection: a propensity score-matched analysis of 12 year single-center experience. Perfusion. 2015;30:243-9.

E40. Kuhn EW, Liakopoulos O, Slottosch I, Deppe AC, Choi YH, Madershahian N et al. Buckberg versus Calafiore cardioplegia in patients with acute coronary syndromes. Thorac Cardiovasc Surg. 2018;66:457-63.

E41. Mourad FA, Fadala MA, Ibrahim AA, Ammar AM, Elnahas YM Elghanam MA, et al. Myocardial protection during CABG: warm blood versus cold crystalloid cardioplegia, is there any difference? J Egypt Soc Cardio Thorac Surg. 2016;24:215-22.

E42. Nardi P, Vacirca SR, Russo M, Colella DF, Bassano C, Scafuri A, et al. Cold crystalloid versus warm blood cardioplegia in patients undergoing aortic valve replacement. J Thorac Dis. 2018;10:1490-9.

E43. Nardi P, Pisano C, Bertoldo F, Vacirca SR, Saitto G, Costantino A, et al. Warm blood cardioplegia versus cold crystalloid cardioplegia for myocardial protection during coronary artery bypass grafting surgery. Cell Death Discov. 2018;4: 23.

E44. Plicner D, Stoliński JJJ, Rzucidlo-Hymczak A, Kapelak B, Undas B, RzucidloHymczak A. Acute-phase proteins and oxidative stress in patients undergoing coronary artery bypass graft: comparison of cardioplegia strategy. Kardiochir Torakochirurgia Pol. 2017;14:16-21. 
E45. Rosu C, Laflamme M, Perrault-Hébert C, Carrier M, Perrault LP. Decreased incidence of low output syndrome with a switch from tepid to cold continuous minimally diluted blood cardioplegia in isolated coronary artery bypass grafting. Interact Cardiovasc Thorac Surg. 2012;15:655-60.

E46. Trescher K, Gleiss A, Boxleitner M, Dietl W, Kassal H, Holzinger C, et al. Short-term clinical outcomes for intermittent cold versus intermittent warm blood cardioplegia in 2200 adult cardiac surgery patients. J Cardiovasc Surg (Torino). 2017;58:105-12.

E47. Zeriouh M, Heider A, Rahmanian PB, Choi YH, Sabashnikov A, Scherner M, et al. Six-years survival and predictors of mortality after CABG using cold vs. warm blood cardioplegia in elective and emergent settings. J Cardiothorac Surg. 2015;10:1-13. 


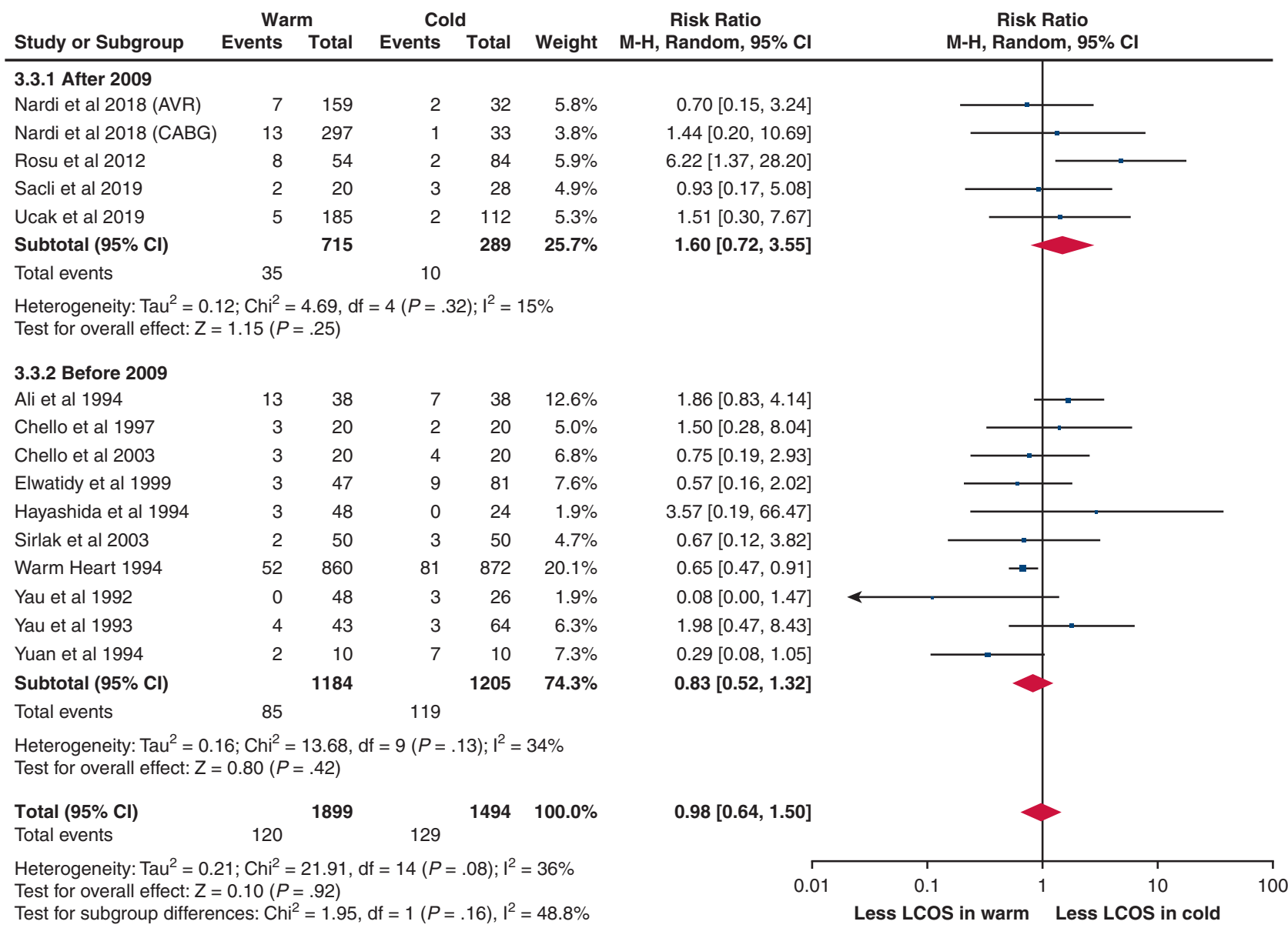

FIGURE E1. Forest plot for low cardiac output syndrome. Subgroup analysis of studies published after Fan and colleagues. ${ }^{\text {E1 }} M-H$, Mantel-Haenszel; $C I$, confidence interval; $A V R$, aortic valve replacement; $C A B G$, coronary artery bypass graft; $L C O S$, low cardiac output syndrome. 


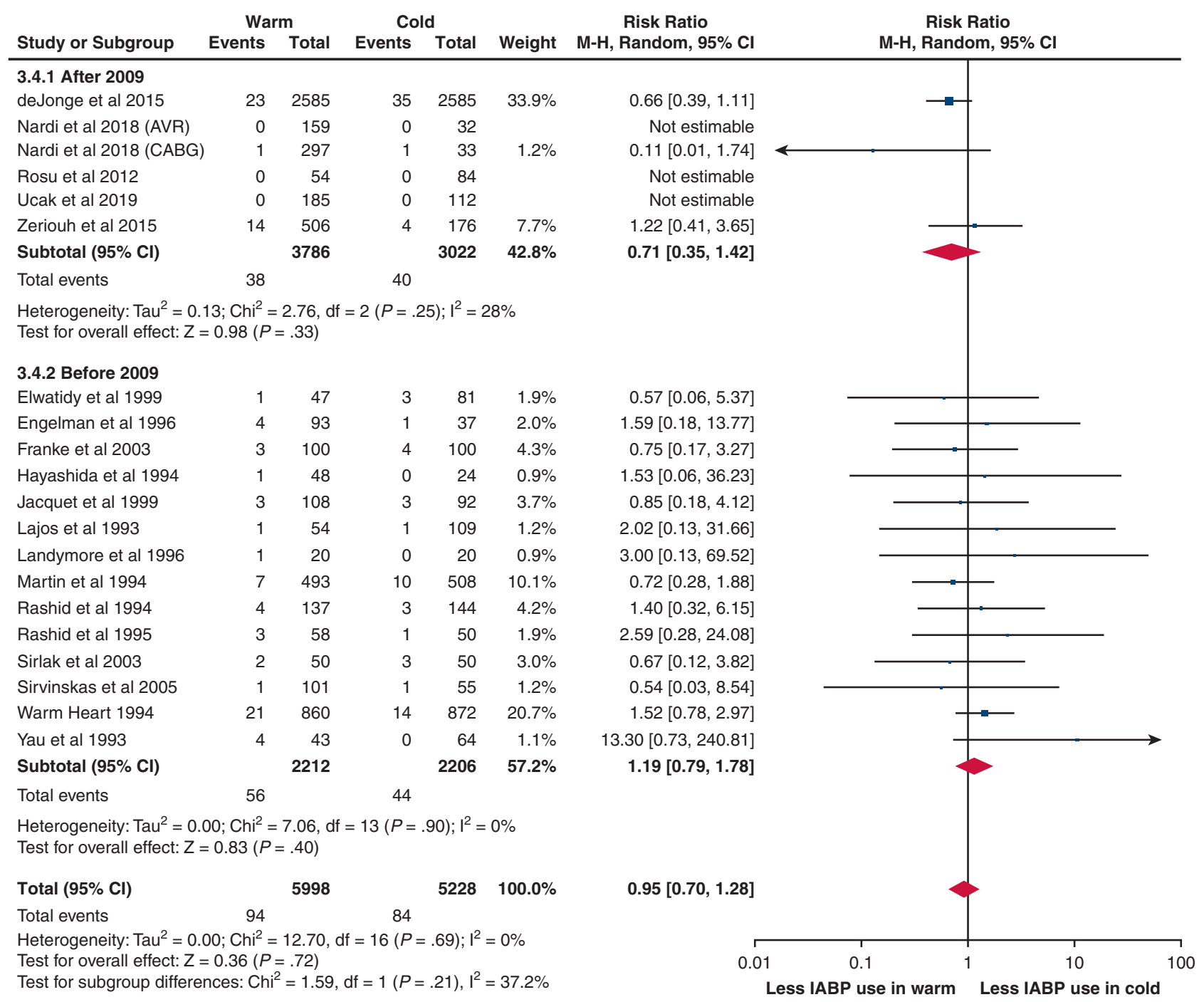

FIGURE E2. Forest plot for intra-aortic balloon pump use. Subgroup analysis of studies published after Fan and colleagues. ${ }^{\text {E1 }} M-H$, Mantel-Haenszel; $C I$, confidence interval; $A V R$, aortic valve replacement; $C A B G$, coronary artery bypass graft; $I A B P$, intra-aortic balloon pump. 
Study or Subgroup

Warm

Cold

Risk Ratio

Events Total Events Total Weight M-H, Random, 95\% C

Risk Ratio

3.5.1 After 2009

Candilio et al 2014

Gaudino et al 2013

Kammerer et al 2010

Kuhn et al 2018

Nardi et al 2018 (AVR)

Rosu et al 2012

Trescher et al 2017

Ucak et al 2019

Subtotal $(95 \% \mathrm{Cl})$

$\begin{array}{rr}0 & \\ 1 & \\ 0 & \\ 3 & 2 \\ 3 & 2 \\ 1 & \\ 12 & 6 \\ 7 & 18 \\ & \\ & \end{array}$

Total events

27

$\begin{array}{rrrr}10 & 0 & 28 & \\ 29 & 0 & 31 & 1.2 \% \\ 52 & 0 & 55 & \\ 212 & 7 & 212 & 7.0 \% \\ 297 & 0 & 33 & 1.4 \% \\ 54 & 2 & 84 & 2.2 \% \\ 610 & 29 & 1578 & 28.1 \% \\ 185 & 3 & 112 & 7.0 \% \\ 1449 & & 2133 & \mathbf{4 7 . 0 \%}\end{array}$

Not estimable

$3.20[0.14,75.55]$

Not estimable

$0.43[0.11,1.64]$

$0.80[0.04,15.14]$

$0.78[0.07,8.37]$

$1.07[0.55,2.08]$

$1.41[0.37,5.35]$

$0.98[0.59,1.64]$

Heterogeneity: $\mathrm{Tau}^{2}=0.00 ; \mathrm{Chi}^{2}=2.42, \mathrm{df}=5(P=.79) ; \mathrm{I}^{2}=0 \%$

Test for overall effect: $Z=0.08(P=.94)$

3.5.2 Before 2009

Chocron et al 2000

Franke et al 2003

Hayashida et al 1994

Jacquet et al 1999

Lajos et al 1993

Martin et al 1994

Pelletier et al 1994

Warm Heart 1994

Yau et al 1993

Subtotal $(95 \% \mathrm{Cl})$

Total events

Heterogeneity: $\mathrm{Tau}^{2}=0.00 ; \mathrm{Chi}^{2}=6.60, \mathrm{df}=8(P=.58) ; \mathrm{I}^{2}=0 \%$

Test for overall effect: $Z=1.37(P=.17)$

Total $(95 \% \mathrm{Cl})$

3300

70

$\begin{array}{rrrr}45 & 1 & 45 & 1.2 \% \\ 100 & 4 & 100 & 7.5 \% \\ 48 & 0 & 24 & 1.5 \% \\ 108 & 2 & 92 & 3.3 \% \\ 54 & 0 & 54 & 1.4 \% \\ 493 & 5 & 508 & 12.4 \% \\ 100 & 2 & 100 & 2.2 \% \\ 860 & 13 & 872 & 22.2 \% \\ 43 & 1 & 64 & 1.2 \% \\ 1851 & & 1859 & 53.0 \%\end{array}$

$0.33[0.01,7.97]$

$1.25[0.35,4.52]$

$3.57[0.19,66.47]$

$0.85[0.12,5.93]$

7.00 [0.37, 132.35]

$3.09[1.13,8.44]$

$0.50[0.05,5.43]$

$1.09[0.52,2.31]$

$0.49[0.02,11.81]$

$1.40[0.86,2.28]$

Total events

$3992 \quad 100.0 \%$

Heterogeneity: $\mathrm{Tau}^{2}=0.00 ; \mathrm{Chi}^{2}=10.00, \mathrm{df}=14(P=.76) ; \mathrm{I}^{2}=0 \%$

Test for overall effect: $Z=0.94(P=.35)$

Test for subgroup differences: $\mathrm{Chi}^{2}=0.99, \mathrm{df}=1(P=.32), \mathrm{I}^{2}=0 \%$
M-H, Random, 95\% Cl

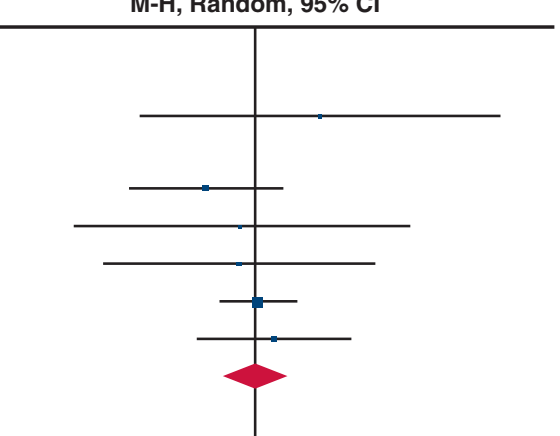

FIGURE E3. Forest plot for stroke. Subgroup analysis of studies published after Fan and colleagues. ${ }^{\text {E1 }} M-H$, Mantel-Haenszel; $C I$, confidence interval. 


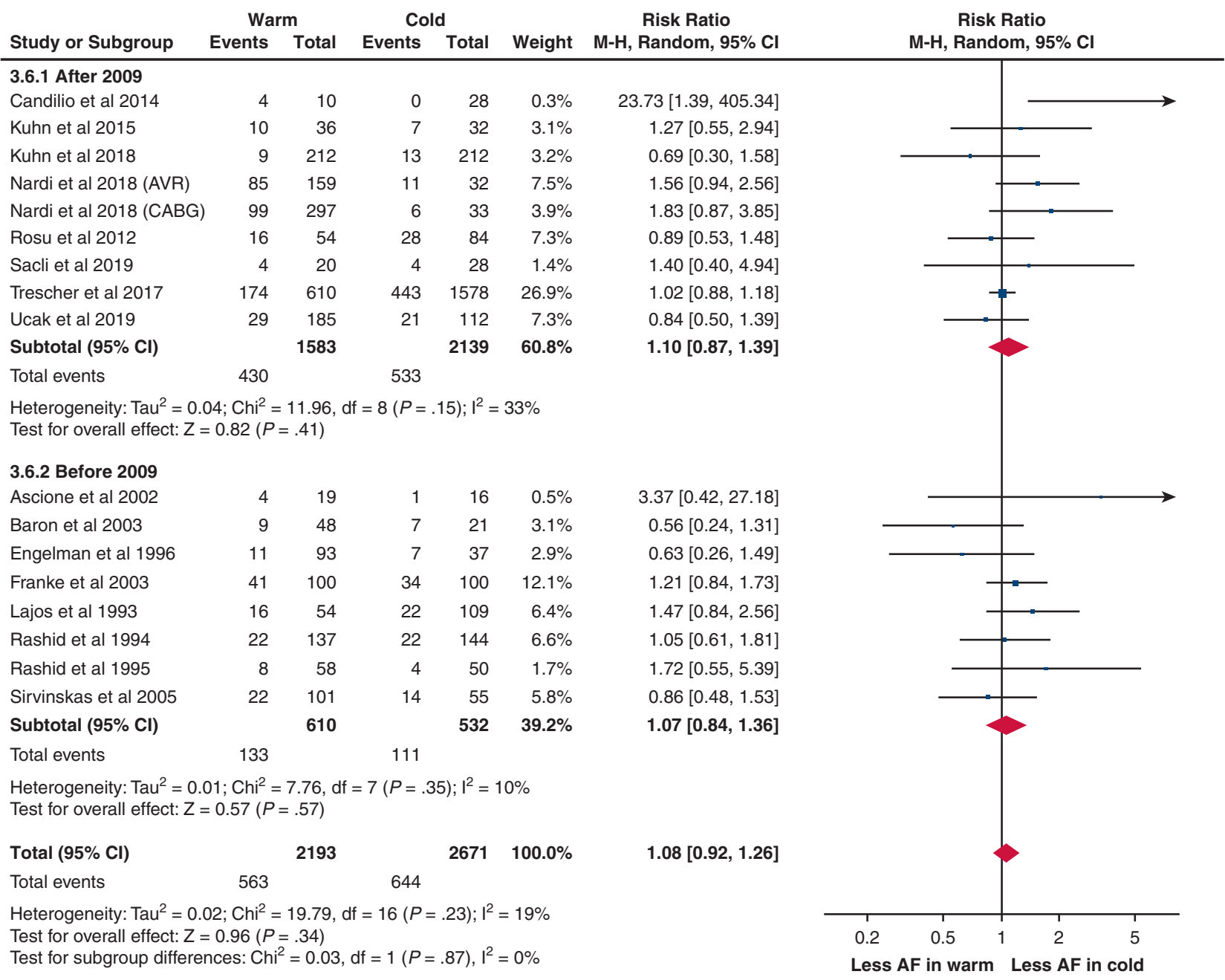

FIGURE E4. Forest plot for new atrial fibrillation. Subgroup analysis of studies published after Fan and colleagues. ${ }^{\mathrm{E} 1} M-H$, Mantel-Haenszel; $C I$, confidence interval; $A V R$, aortic valve replacement; $C A B G$, coronary artery bypass graft; $A F$, atrial fibrillation.

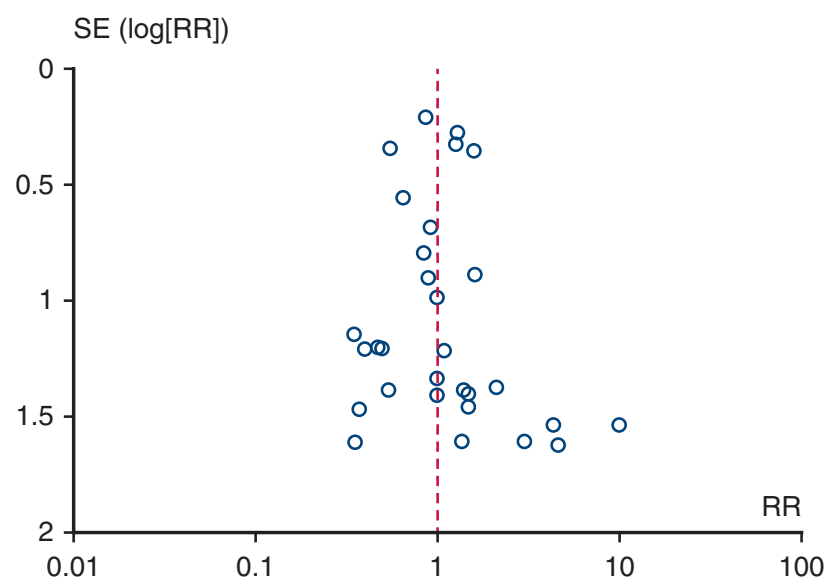

FIGURE E5. Funnel plot for mortality. $S E$, Standard error; $R R$, relative risk.

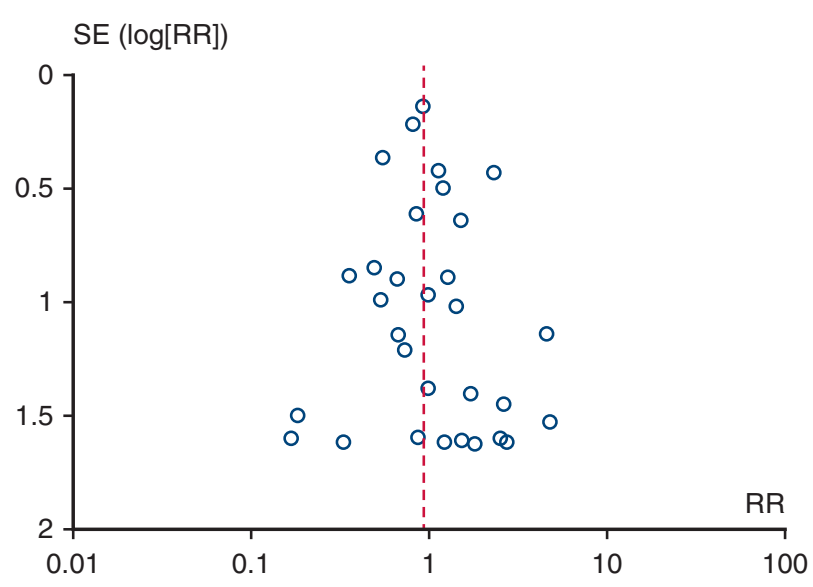

FIGURE E6. Funnel plot for myocardial infarction. SE, Standard error; $R R$, relative risk. 


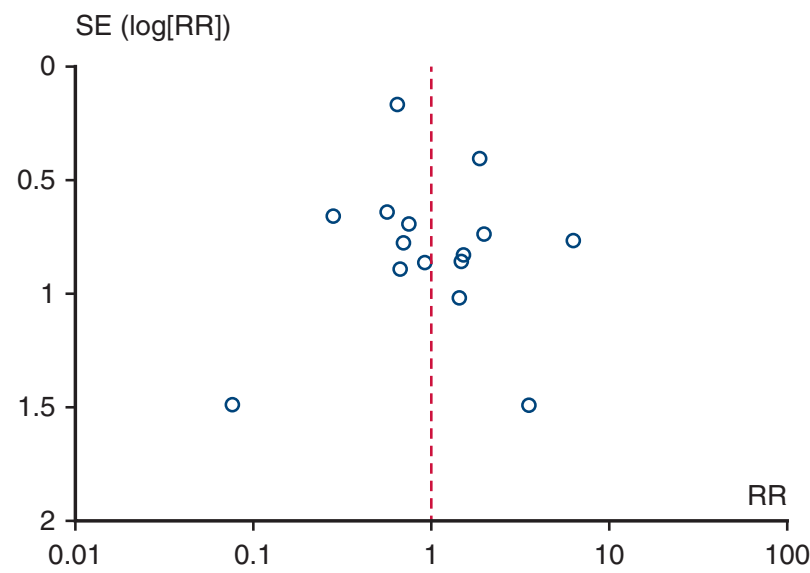

FIGURE E7. Funnel plot for low cardiac output syndrome. SE, Standard error; $R R$, relative risk.

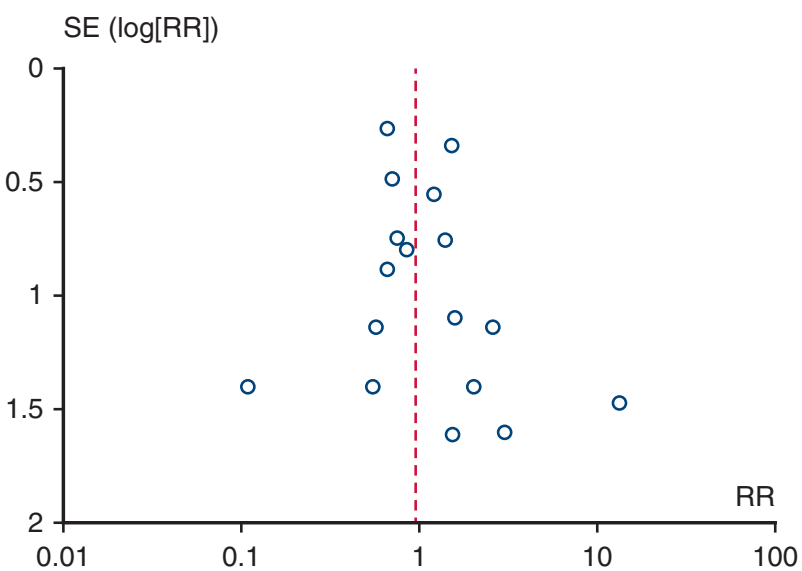

FIGURE E8. Funnel plot for intra-aortic balloon pump use. $S E$, Standard error; $R R$, relative risk.

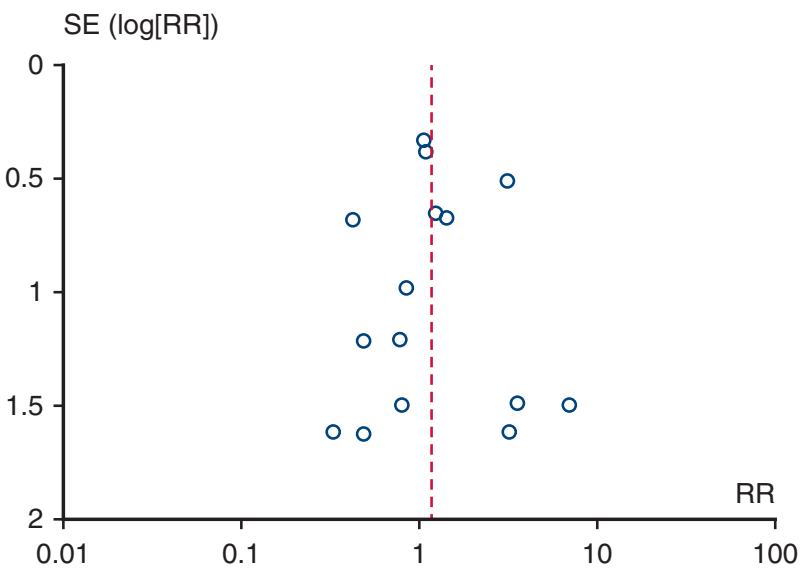

FIGURE E9. Funnel plot for stroke. $S E$, Standard error; $R R$, relative risk.

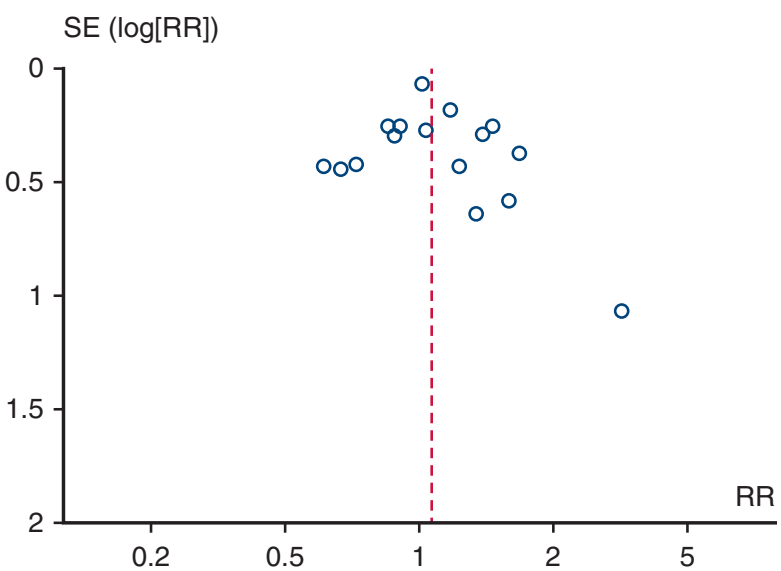

FIGURE E10. Funnel plot for new atrial fibrillation. $S E$, Standard error; $R R$, relative risk.

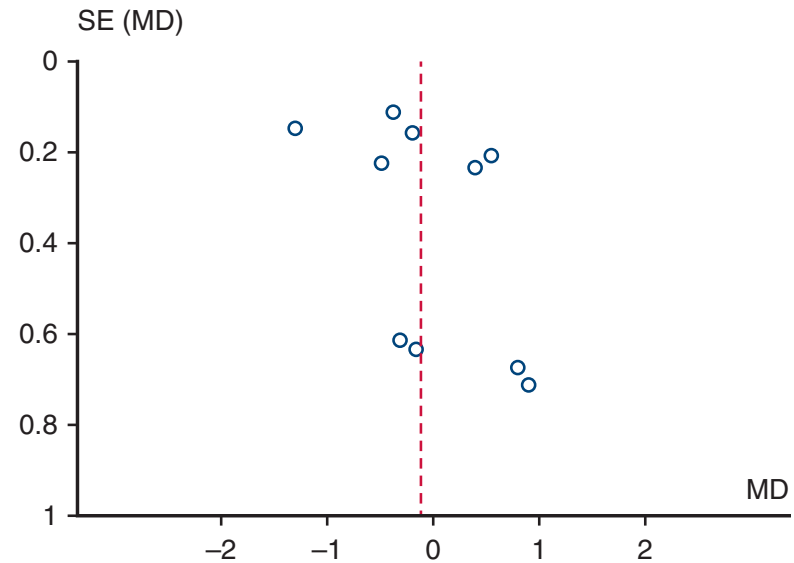

FIGURE E11. Funnel plot for intensive care unit length of stay. SE, Standard error; $M D$, mean difference. 


\begin{tabular}{|c|c|c|c|c|c|c|c|c|c|}
\hline Authors & $\begin{array}{l}\text { Was the study } \\
\text { described as } \\
\text { randomized? } \\
\text { Yes: } 1 \text { mark } \\
\text { No: } 0 \text { mark }\end{array}$ & $\begin{array}{c}\text { Was the } \\
\text { method of } \\
\text { randomization } \\
\text { appropriate? } \\
\text { Yes: } 1 \text { mark } \\
\text { No: }-1 \text { mark } \\
\text { Not described: } \\
\text { 0 mark }\end{array}$ & $\begin{array}{c}\text { Was the study } \\
\text { described as } \\
\text { blinded? } \\
\text { Double-blind: } 1 \\
\text { mark } \\
\text { Single-blind: } 0.5 \\
\text { mark } \\
\text { No: } 0 \text { mark }\end{array}$ & $\begin{array}{c}\text { Was the method } \\
\text { of blinding } \\
\text { appropriate? } \\
\text { Yes: } 1 \text { mark } \\
\text { No: -1 mark } \\
\text { Not described: } \\
\text { 0 mark }\end{array}$ & $\begin{array}{l}\text { Was there a } \\
\text { description of } \\
\text { withdrawals and } \\
\text { dropouts? } \\
\text { Yes: } 1 \text { mark } \\
\text { No: } 0 \text { mark }\end{array}$ & $\begin{array}{c}\text { Was there a } \\
\text { clear description } \\
\text { of the inclusion/ } \\
\text { exclusion } \\
\text { criteria? } \\
\text { Yes: } 1 \text { mark } \\
\text { No: } 0 \text { mark }\end{array}$ & $\begin{array}{l}\text { Was the method } \\
\text { used to assess } \\
\text { adverse effects } \\
\text { described? } \\
\text { Yes: } 1 \text { mark } \\
\text { No: } 0 \text { mark }\end{array}$ & $\begin{array}{l}\text { Were the } \\
\text { methods of } \\
\text { statistical } \\
\text { analysis } \\
\text { described? } \\
\text { Yes: } 1 \text { mark } \\
\text { No: } 0 \text { mark }\end{array}$ & $\begin{array}{c}\text { Total } \\
\text { score } \\
\text { (out of } 8 \text { ) }\end{array}$ \\
\hline Ali et al $1994^{\mathrm{E} 2}$ & 1 & -1 & 0 & 0 & 0 & 1 & 1 & 1 & 3 \\
\hline $\begin{array}{l}\text { Ascione et al } \\
2002^{\mathrm{E3}}\end{array}$ & 1 & 0 & 0 & 0 & 1 & 1 & 1 & 1 & 5 \\
\hline Baron et al $2003^{\mathrm{E} 4}$ & 1 & 0 & 0 & 0 & 0 & 0 & 1 & 1 & 3 \\
\hline Chello et al $1997^{\mathrm{E} 5}$ & 1 & -1 & 0 & 0 & 0 & 0 & 1 & 1 & 2 \\
\hline Chello et al $2003^{\mathrm{E} 6}$ & 1 & 0 & 0 & 0 & 1 & 0 & 1 & 1 & 4 \\
\hline $\begin{array}{l}\text { Chocron et al } \\
2000^{\mathrm{E}}\end{array}$ & 1 & 1 & 0 & 0 & 1 & 1 & 1 & 1 & 6 \\
\hline Curtis et al $1996^{\mathrm{E} 8}$ & 1 & 0 & 0 & 0 & 1 & 0 & 1 & 1 & 4 \\
\hline Dar et al $2005^{\mathrm{E} 9}$ & 1 & 0 & 0 & 0 & 0 & 1 & 1 & 1 & 4 \\
\hline $\begin{array}{c}\text { Elwatidy et al } \\
1999^{\mathrm{E} 10}\end{array}$ & 1 & 0 & 0 & 0 & 1 & 0 & 1 & 1 & 4 \\
\hline $\begin{array}{l}\text { Engelman et al } \\
\qquad 1996^{\text {E11 }}\end{array}$ & 1 & 1 & 1 & 1 & 1 & 1 & 1 & 1 & 8 \\
\hline $\begin{array}{c}\text { Franke et al } \\
2003^{\mathrm{E} 12}\end{array}$ & 1 & 1 & 0 & 0 & 1 & 1 & 1 & 1 & 6 \\
\hline $\begin{array}{l}\text { Gaudino et al } \\
2013^{\mathrm{E} 13}\end{array}$ & 1 & 1 & 1 & 0 & 1 & 1 & 1 & 1 & 7 \\
\hline $\begin{array}{l}\text { Hayashida et al } \\
1994^{\text {E14 }}\end{array}$ & 1 & 1 & 0 & 0 & 1 & 0 & 0 & 1 & 4 \\
\hline $\begin{array}{l}\text { Hayashida et al } \\
1995^{\text {E15 }}\end{array}$ & 1 & 1 & 0 & 0 & 0 & 0 & 1 & 1 & 4 \\
\hline $\begin{array}{l}\text { Honkonen et al } \\
1997^{\mathrm{E} 16}\end{array}$ & 1 & 0 & 0 & 0 & 0 & 1 & 1 & 1 & 4 \\
\hline $\begin{array}{c}\text { Isomura et al } \\
1995^{\mathrm{E} 17}\end{array}$ & 1 & 0 & 0 & 0 & 0 & 0 & 1 & 1 & 3 \\
\hline $\begin{array}{c}\text { Jacquet et al } \\
1999^{\mathrm{E} 18}\end{array}$ & 1 & 0 & 0 & 0 & 1 & 1 & 1 & 1 & 5 \\
\hline $\begin{array}{c}\text { Kammerer et al } \\
2010^{\mathrm{E} 19}\end{array}$ & 1 & 0 & 0 & 0 & 0 & 1 & 1 & 1 & 4 \\
\hline
\end{tabular}




\begin{tabular}{|c|c|c|c|c|c|c|c|c|c|}
\hline Authors & $\begin{array}{l}\text { Was the study } \\
\text { described as } \\
\text { randomized? } \\
\text { Yes: } 1 \text { mark } \\
\text { No: } 0 \text { mark }\end{array}$ & $\begin{array}{c}\text { Was the } \\
\text { method of } \\
\text { randomization } \\
\text { appropriate? } \\
\text { Yes: } 1 \text { mark } \\
\text { No: }-1 \text { mark } \\
\text { Not described: } \\
\text { 0 mark }\end{array}$ & $\begin{array}{l}\text { Was the study } \\
\text { described as } \\
\text { blinded? } \\
\text { Double-blind: } 1 \\
\text { mark } \\
\text { Single-blind: } 0.5 \\
\text { mark } \\
\text { No: } 0 \text { mark }\end{array}$ & $\begin{array}{c}\text { Was the method } \\
\text { of blinding } \\
\text { appropriate? } \\
\text { Yes: } 1 \text { mark } \\
\text { No: }-1 \text { mark } \\
\text { Not described: } \\
\text { 0 mark }\end{array}$ & $\begin{array}{l}\text { Was there a } \\
\text { description of } \\
\text { withdrawals and } \\
\text { dropouts? } \\
\text { Yes: } 1 \text { mark } \\
\text { No: } 0 \text { mark }\end{array}$ & $\begin{array}{c}\text { Was there a } \\
\text { clear description } \\
\text { of the inclusion/ } \\
\text { exclusion } \\
\text { criteria? } \\
\text { Yes: } 1 \text { mark } \\
\text { No: } 0 \text { mark }\end{array}$ & $\begin{array}{l}\text { Was the method } \\
\text { used to assess } \\
\text { adverse effects } \\
\text { described? } \\
\text { Yes: } 1 \text { mark } \\
\text { No: } 0 \text { mark }\end{array}$ & $\begin{array}{l}\text { Were the } \\
\text { methods of } \\
\text { statistical } \\
\text { analysis } \\
\text { described? } \\
\text { Yes: } 1 \text { mark } \\
\text { No: } 0 \text { mark }\end{array}$ & $\begin{array}{c}\text { Total } \\
\text { score } \\
\text { (out of 8) }\end{array}$ \\
\hline Kuhn et al $2015^{\text {E20 }}$ & 1 & 1 & 1 & 0 & 1 & 1 & 1 & 1 & 7 \\
\hline Lajos et al $1993^{\mathrm{E} 21}$ & 1 & -1 & 0 & 0 & 1 & 0 & 1 & 1 & 3 \\
\hline $\begin{array}{l}\text { Landymore et al } \\
1996^{\mathrm{E} 22}\end{array}$ & 1 & 1 & 0 & 0 & 0 & 1 & 1 & 1 & 5 \\
\hline $\begin{array}{l}\text { Maccherini et al } \\
1995^{\mathrm{E} 23}\end{array}$ & 1 & 0 & 0 & 0 & 0 & 0 & 1 & 0 & 2 \\
\hline $\begin{array}{c}\text { Martin et al } \\
1994^{\mathrm{E} 24}\end{array}$ & 1 & 1 & 0 & 0 & 1 & 0 & 0 & 1 & 4 \\
\hline $\begin{array}{c}\text { Pelletier et al } \\
1994^{\mathrm{E25}}\end{array}$ & 1 & 1 & 0.5 & 1 & 0 & 1 & 1 & 1 & 6.5 \\
\hline $\begin{array}{c}\text { Pepper et al } \\
1995^{\mathrm{E} 26}\end{array}$ & 1 & 0 & 0.5 & 0 & 0 & 0 & 1 & 1 & 3.5 \\
\hline $\begin{array}{c}\text { Rashid et al } \\
1994^{\mathrm{E} 27}\end{array}$ & 1 & -1 & 0 & 0 & 0 & 0 & 1 & 1 & 2 \\
\hline $\begin{array}{c}\text { Rashid et al } \\
1995^{\mathrm{E} 28}\end{array}$ & 1 & -1 & 0 & 0 & 0 & 0 & 1 & 1 & 2 \\
\hline Saclı et al $2019^{\mathrm{E} 29}$ & 1 & -1 & 0 & 0 & 0 & 1 & 1 & 1 & 3 \\
\hline $\begin{array}{c}\text { Şirlak et al } \\
2003^{\mathrm{E} 30}\end{array}$ & 1 & 0 & 0 & 0 & 1 & 1 & 1 & 1 & 5 \\
\hline $\begin{array}{l}\text { Sirvinskas et al } \\
2005^{\mathrm{E} 31}\end{array}$ & 1 & 1 & 0 & 0 & 1 & 1 & 1 & 1 & 6 \\
\hline $\begin{array}{l}\text { The Warm Heart } \\
\text { Investigator } \\
1994^{\mathrm{E} 32}\end{array}$ & 1 & 1 & 0.5 & 0 & 1 & 1 & 1 & 1 & 6.5 \\
\hline Ucak et al $2019^{\mathrm{E} 33}$ & 1 & 1 & 0 & 0 & 0 & 1 & 1 & 1 & 5 \\
\hline Yau et al $1992^{\mathrm{E} 34}$ & 1 & 1 & 0 & 0 & 0 & 0 & 0 & 1 & 3 \\
\hline Yau et al $1993^{\mathrm{E} 35}$ & 1 & 1 & 0 & 0 & 1 & 0 & 1 & 1 & 5 \\
\hline Yang et al $1994^{\mathrm{E} 36}$ & 1 & 0 & 0 & 0 & 0 & 0 & 0 & 1 & 2 \\
\hline
\end{tabular}


representativeness

is the exposed

group of

the typical

Does the

study clearly

person in the

show that

community?

of the

Truly/somewhat

representative

with the

of the average in the

cohort

Same

outcome were

community:

community as

Was exposure

status ascertained

in a way that

Does the study

adequately dea

Assessment of

outcome

Adequacy

prevents/minimizes with potential Independent or blind

of FU of

misclassification of

confounders? assessment stated in

cohorts

exposure status? Study controls for the paper, or

Complete

Secure or unbiased most important confirmation of the

record/structured confounding factor:

outcome by

FU/subjects

excluded from

interview: 1 mark

1 mark

reference to secure clinical judgement, unlikely to

cohort: 1 mark

Written self-report, Study further

records/record was FU long enough introduce

Select,

Different

Yes: 1 mark

unrepresentative

source, no

interview without

controls for any

linkage (ICD): 1 for outcomes to bias: 1 mark

validated additional potential

mark

occur?

No Total

questionnaire, no confounding factor: Self-report, no Yes: 1 mark description: score

\begin{tabular}{|c|c|c|c|c|c|c|c|c|c|}
\hline Authors & No: 0 mark & -0 mark & 0 mark & description: 0 mark & 1 mark & descriptions: 0 mark & No: 0 mark & 0 mark & (out of 9) \\
\hline Raza Baig & 1 & 1 & 1 & 1 & 2 & 1 & 1 & 1 & 9 \\
\hline
\end{tabular}

et al $2015^{\mathrm{E} 37}$

\begin{tabular}{|c|c|c|c|c|c|c|c|c|c|}
\hline $\begin{array}{l}\text { Candilio } \\
\text { et al } 2014^{\mathrm{E} 38}\end{array}$ & 1 & 1 & 1 & 1 & 2 & 1 & 1 & 1 & 9 \\
\hline $\begin{array}{l}\text { De Jonge } \\
\text { et al } 2015^{\mathrm{E} 39}\end{array}$ & 0 & 1 & 1 & 1 & 2 & 1 & 1 & 1 & 8 \\
\hline $\begin{array}{l}\text { Kuhn } \\
\text { et al } 2018^{\mathrm{E} 40}\end{array}$ & 0 & 1 & 1 & 1 & 2 & 1 & 1 & 1 & 8 \\
\hline $\begin{array}{l}\text { Mourad } \\
\quad \text { et al } 2016^{\mathrm{E} 41}\end{array}$ & 1 & 1 & 1 & 1 & 2 & 1 & 1 & 1 & 9 \\
\hline $\begin{array}{l}\text { Nardi } \\
\text { et al } 2018^{\mathrm{E} 42}\end{array}$ & 0 & 1 & 1 & 1 & 2 & 1 & 1 & 1 & 8 \\
\hline $\begin{array}{l}\text { Nardi } \\
\quad \text { et al } 2018^{\mathrm{E} 43}\end{array}$ & 0 & 1 & 1 & 1 & 2 & 1 & 1 & 1 & 8 \\
\hline $\begin{array}{l}\text { Plicner } \\
\text { et al } 2017^{\mathrm{E} 44}\end{array}$ & 1 & 1 & 1 & 1 & 2 & 1 & 1 & 1 & 9 \\
\hline $\begin{array}{l}\text { Rosu } \\
\quad \text { et al } 2012^{\mathrm{E} 45}\end{array}$ & 0 & 1 & 1 & 1 & 2 & 1 & 1 & 1 & 8 \\
\hline $\begin{array}{l}\text { Trescher } \\
\text { et al } 2017^{\mathrm{E} 46}\end{array}$ & 0 & 1 & 1 & 1 & 2 & 1 & 1 & 1 & 8 \\
\hline $\begin{array}{l}\text { Zeriouh } \\
\text { et al } 2015^{\mathrm{E} 47}\end{array}$ & 1 & 1 & 1 & 1 & 2 & 1 & 1 & 1 & 9 \\
\hline
\end{tabular}

ICD, International Classification of Diseases; FU, follow-up 
TABLE E3. Baseline characteristics of patients included after previous meta-analysis

\begin{tabular}{lccr}
\hline & OR or WMD [95\% CI] & P value & Reporting studies, n (\%) \\
\hline Age & WMD $0.01[-0.43,0.45]$ & .98 & $13(81.25 \%)$ \\
Male & OR $0.74[0.62-0.89]$ & .001 & $15(93.75 \%)$ \\
Hypertension & OR $1.05[0.86-1.29]$ & .63 & $11(68.75 \%)$ \\
DM & OR $1.08[0.97-1.19]$ & .15 & $11(68.75 \%)$ \\
LVEF & WMD $-0.15[-1.64,1.34]$ & .85 & $10(62.50 \%)$ \\
\hline
\end{tabular}

$O R$, Odds ratio; $W M D$, weighted mean difference; $C I$, confidence interval; $D M$, diabetes mellitus; $L V E F$, left ventricular ejection fraction.

TABLE E4. Pairing table

\begin{tabular}{|c|c|c|c|c|c|c|c|c|c|c|c|}
\hline Author & Year & Mortality & MI & LCOS & $\begin{array}{c}\text { IABP } \\
\text { use }\end{array}$ & Stroke & New AF & AKI & Hospital LOS & ICU LOS & $\begin{array}{c}\text { Risk of bias -MJS (/8) } \\
\text { NOS (/9) }\end{array}$ \\
\hline Ali et $\mathrm{a}^{\mathrm{E} 2}$ & 1994 & & $v$ & $\nu$ & & & & & & & $3 / 8$ \\
\hline Ascione et $\mathrm{al}^{\mathrm{E} 3}$ & 2002 & & $\nu$ & & & & $\boldsymbol{r}$ & & & & $5 / 8$ \\
\hline Raza Baig et $\mathrm{l}^{\mathrm{E} 37}$ & 2015 & & $レ$ & & & & & & & & $9 / 9$ \\
\hline Baron et $\mathrm{al}^{\mathrm{E} 4}$ & 2003 & $\boldsymbol{r}$ & & & & & 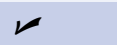 & & & & $3 / 8$ \\
\hline Candilio et $\mathrm{al}^{\mathrm{E} 38}$ & 2014 & $\boldsymbol{\nu}$ & 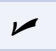 & & & $\boldsymbol{\nu}$ & 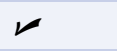 & レ & 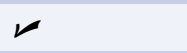 & $\boldsymbol{\nu}$ & $9 / 9$ \\
\hline Chello et $\mathrm{al}^{\mathrm{E5}}$ & 1997 & & & $\nu$ & & & & & & & $2 / 8$ \\
\hline Chello et al ${ }^{\mathrm{E} 6}$ & 2003 & & & $\nu$ & & & & & & & $4 / 8$ \\
\hline Chocron et $\mathrm{al}^{\mathrm{E} 7}$ & 2000 & & $\nu$ & & & $\nu$ & & & & & $6 / 8$ \\
\hline Curtis et $\mathrm{al}^{\mathrm{E} 8}$ & 1996 & レ & $\nu$ & & & & & & & & $4 / 8$ \\
\hline De Jonge et $\mathrm{al}^{\mathrm{E} 39}$ & 2015 & $\nu$ & & & $\nu$ & & & & & $\nu$ & $8 / 9$ \\
\hline Elwatidy et al ${ }^{\mathrm{E} 10}$ & 1999 & & $\nu$ & レ & レ & & & & & & $4 / 8$ \\
\hline Engelman et al ${ }^{\mathrm{E} 11}$ & 1996 & & $\nu$ & & レ & & $\boldsymbol{v}$ & & & & $8 / 8$ \\
\hline Franke et $\mathrm{al}^{\mathrm{E} 12}$ & 2003 & r & $\nu$ & & レ & レ & $\nu$ & & & & $6 / 8$ \\
\hline Gaudino et $\mathrm{al}^{\mathrm{E} 13}$ & 2013 & 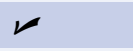 & & & & レ & & & $\boldsymbol{V}$ & $\boldsymbol{\nu}$ & $7 / 8$ \\
\hline Hayashida et $\mathrm{al}^{\mathrm{E} 14}$ & 1994 & & $\nu$ & $\nu$ & レ & レ & & & & & $4 / 8$ \\
\hline Hayashida et al ${ }^{\mathrm{E} 15}$ & 1995 & & $\nu$ & & & & & & & & $4 / 8$ \\
\hline Honkonen et $\mathrm{al}^{\mathrm{E} 16}$ & 1997 & & 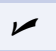 & & & & & & & & $4 / 8$ \\
\hline Isomura et al ${ }^{\mathrm{E} 17}$ & 1995 & & $\nu$ & & & & & & & & $3 / 8$ \\
\hline 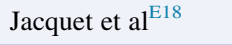 & 1999 & $\boldsymbol{r}$ & $\nu$ & & $\boldsymbol{V}$ & $\boldsymbol{V}$ & & & & & $5 / 8$ \\
\hline Kammerer et al ${ }^{\mathrm{E} 19}$ & 2010 & $\boldsymbol{\nu}$ & & & & 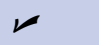 & & & & & $4 / 8$ \\
\hline Kuhn et al ${ }^{\mathrm{E} 20}$ & 2015 & $r$ & & & & & $r$ & & & v & $7 / 8$ \\
\hline Kuhn et $\mathrm{al}^{\mathrm{E} 40}$ & 2018 & $\boldsymbol{\nu}$ & & & & $\nu$ & 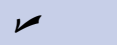 & & 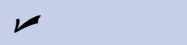 & & $8 / 9$ \\
\hline Lajos et al ${ }^{\mathrm{E} 21}$ & 1993 & $\boldsymbol{V}$ & $\checkmark$ & & レ & レ & $\boldsymbol{v}$ & & & & $3 / 8$ \\
\hline Landymore et al ${ }^{\mathrm{E} 22}$ & 1996 & $\nu$ & $\nu$ & & $\nu$ & & & & & & $5 / 8$ \\
\hline Maccherini et a ${ }^{\mathrm{E} 23}$ & 1995 & $\boldsymbol{V}$ & & & & & & & & & $2 / 8$ \\
\hline Martin et al ${ }^{\mathrm{E} 24}$ & 1994 & $\boldsymbol{V}$ & $\checkmark$ & & $\boldsymbol{V}$ & $\boldsymbol{V}$ & & & & & $4 / 8$ \\
\hline Mourad et al ${ }^{\mathrm{E} 41}$ & 2016 & & 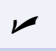 & & & & & & & $\boldsymbol{v}$ & $9 / 9$ \\
\hline Nardi et $\mathrm{al}^{\mathrm{E} 42}$ & 2018 & V & $\nu$ & V & レ & レ & $r$ & V & $r$ & V & $8 / 9$ \\
\hline Nardi et $\mathrm{al}^{\mathrm{E} 43}$ & 2018 & $\boldsymbol{V}$ & $\checkmark$ & $\boldsymbol{v}$ & レ & & $\boldsymbol{r}$ & $\boldsymbol{\nu}$ & $\boldsymbol{v}$ & $\boldsymbol{\nu}$ & $8 / 9$ \\
\hline Pelletier et al ${ }^{\mathrm{E} 25}$ & 1994 & $\boldsymbol{r}$ & $v$ & & & レ & & & & & $6.5 / 8$ \\
\hline Pepper et $\mathrm{al}^{\mathrm{E} 26}$ & 1995 & レ & & & & & & & & & $3.5 / 8$ \\
\hline Plicner et $\mathrm{al}^{\mathrm{E} 44}$ & 2017 & $r$ & $\nu$ & & & & & & & & $9 / 9$ \\
\hline Rashid et al $^{\mathrm{E} 27}$ & 1994 & $\boldsymbol{v}$ & $\nu$ & & レ & & $r$ & & & & $2 / 8$ \\
\hline Rashid et $\mathrm{al}^{\mathrm{E} 28}$ & 1995 & $\boldsymbol{r}$ & $v$ & & $\boldsymbol{V}$ & & $r$ & & & & $2 / 8$ \\
\hline Rosu et $\mathrm{al}^{\mathrm{E} 45}$ & 2012 & $\boldsymbol{\nu}$ & $\nu$ & $\nu$ & $\nu$ & $\nu$ & $v$ & $\nu$ & $\nu$ & $\nu$ & $8 / 9$ \\
\hline
\end{tabular}


TABLE E4. Continued

\begin{tabular}{|c|c|c|c|c|c|c|c|c|c|c|c|}
\hline Author & Year & Mortality & MI & LCOS & $\begin{array}{c}\text { IABP } \\
\text { use }\end{array}$ & Stroke & New AF & AKI & Hospital LOS & ICU LOS & $\begin{array}{c}\text { Risk of bias -MJS (/8) } \\
\text { NOS (/9) }\end{array}$ \\
\hline Sacli et al ${ }^{\mathrm{E} 29}$ & 2019 & $\nu$ & & $\nu$ & & & $\nu$ & $\nu$ & $\nu$ & $\nu$ & $3 / 8$ \\
\hline Şirlak et al ${ }^{\mathrm{E} 30}$ & 2003 & & & $\nu$ & $\boldsymbol{\nu}$ & & & & & & $5 / 8$ \\
\hline Sirvinskas et $\mathrm{al}^{\mathrm{E} 31}$ & 2005 & $\nu$ & $\nu$ & & $\nu$ & & $\nu$ & & & & $6 / 8$ \\
\hline $\begin{array}{l}\text { The Warm Heart } \\
\text { Investigators }^{\mathrm{E} 32}\end{array}$ & 1994 & $\boldsymbol{V}$ & レ & & & $\nu$ & $\boldsymbol{V}$ & レ & & & $6.5 / 8$ \\
\hline Trescher et $\mathrm{al}^{\mathrm{E} 46}$ & 2017 & $\nu$ & $\nu$ & $\nu$ & レ & レ & $\nu$ & $\nu$ & レ & $\nu$ & $8 / 9$ \\
\hline Ucak et al ${ }^{\mathrm{E} 33}$ & 2019 & $\nu$ & $\nu$ & $\nu$ & $\nu$ & $\nu$ & & & & & $5 / 8$ \\
\hline Yau et $\mathrm{al}^{\mathrm{E} 34}$ & 1992 & レ & V & $\nu$ & & & & & & & $3 / 8$ \\
\hline Yau et $\mathrm{al}^{\mathrm{E} 35}$ & 1993 & レ & $\nu$ & $\nu$ & $\nu$ & $\nu$ & & & & & $5 / 8$ \\
\hline Yang et $\mathrm{al}^{\mathrm{E} 36}$ & 1994 & $\boldsymbol{v}$ & & $v$ & & & & & & & $2 / 8$ \\
\hline Zeriouh et al ${ }^{\mathrm{E} 47}$ & 2015 & レ & $\nu$ & & $\nu$ & & & & $\nu$ & $\nu$ & $9 / 9$ \\
\hline
\end{tabular}

$M I$, Myocardial infarction; $L C O S$, low cardiac output syndrome; $I A B P$, intra-aortic balloon pump; $A F$, atrial fibrillation; $A K I$, acute kidney injury; $I C U$, intensive care unit; $L O S$, length of stay; MJS, modified Jadad scale; NOS, Newcastle-Ottawa Quality Assessment Scale.

TABLE E5. Summary of primary outcomes since publication of previous meta-analysis

\begin{tabular}{lccc}
\hline & RR [95\% CI] & $\boldsymbol{P}$ value & $\begin{array}{c}\text { Subgroup differences }(\boldsymbol{P} \\
\text { value })\end{array}$ \\
\hline Mortality & $1.09[0.85-1.41]$ & .50 & .16 \\
MI & $1.05[0.71-1.57]$ & .80 & .54 \\
LCOS & $1.60[0.72-3.55]$ & .25 & .16 \\
IABP use & $0.71[0.35-1.42]$ & .33 & .21 \\
Stroke & $0.98[0.59-1.64]$ & .94 & .32 \\
New AF & $1.10[0.87-1.39]$ & .41 & .87 \\
\hline$R R$, Retion & \\
\hline
\end{tabular}

$R R$, Relative risk; $C I$, confidence interval; $M I$, myocardial infarction; $L C O S$, low cardiac output syndrome; $I A B P$, intra-aortic balloon pump; $A F$, atrial fibrillation.

TABLE E6. Summary of primary and secondary outcomes from low risk of bias studies

\begin{tabular}{|c|c|c|c|}
\hline & RR or WMD $[95 \% \mathrm{CI}]$ & $P$ value & $\begin{array}{c}\text { Subgroup differences ( } P \\
\text { value) }\end{array}$ \\
\hline Mortality & RR 1.00 [0.79-1.27] & .99 & .85 \\
\hline MI & RR 0.92 [0.76-1.12] & .42 & .67 \\
\hline LCOS & RR 1.19 [0.61-2.31] & .61 & .45 \\
\hline IABP use & RR 0.95 [0.65-1.39] & .80 & .89 \\
\hline Stroke & RR 0.98 [0.67-1.44] & .92 & .01 \\
\hline New AF & RR 1.07 [0.89-1.29] & .49 & .75 \\
\hline AKI & RR 0.97 [0.57-1.66] & .92 & .70 \\
\hline Hospital LOS & WMD $-0.84[-1.59,-0.10]$ & .03 & .07 \\
\hline ICU LOS & WMD $-0.18[-0.63,0.27]$ & .43 & .15 \\
\hline
\end{tabular}

$R R$, Relative risk; $W M D$, weighted mean difference; $C I$, confidence interval; $M I$, myocardial infarction; $L C O S$, low cardiac output syndrome; $I A B P$, intra-aortic balloon pump; $A F$, atrial fibrillation; $A K I$, acute kidney injury; $L O S$, length of stay; $I C U$, intensive care unit. 
TABLE E7. Summary of primary and secondary outcomes from studies with coronary artery bypass graft performed

\begin{tabular}{lccc}
\hline & RR or WMD [95\% CI] & P value & $\begin{array}{c}\text { Subgroup differences }(\boldsymbol{P} \\
\text { value })\end{array}$ \\
\hline Mortality & RR $0.95[0.74-1.21]$ & .65 & .66 \\
MI & RR $0.88[0.73-1.07]$ & .21 & .08 \\
LCOS & RR $1.00[0.62-1.61]$ & 1.00 & .12 \\
IABP use & RR $0.95[0.70-1.28]$ & .72 & N/A \\
Stroke & RR $1.22[0.80-1.87]$ & .35 & .77 \\
New AF & RR $1.05[0.86-1.28]$ & .64 & .50 \\
AKI & RR $0.84[0.40,1.76]$ & .65 & .51 \\
Hospital LOS & WMD $-0.69[-1.66,0.28]$ & .16 & .18 \\
ICU LOS & WMD $-0.04[-0.56,0.48]$ & .89 & .43 \\
\hline$R R$, Rivi
\end{tabular}

$R R$, Relative risk; $W M D$, weighted mean difference; $C I$, confidence interval; $M I$, myocardial infarction; $L C O S$, low cardiac output syndrome; IABP, intra-aortic balloon pump $N / A$, not available; $A F$, atrial fibrillation; $A K I$, acute kidney injury; $L O S$, length of stay; $I C U$, intensive care unit.

TABLE E8. Summary of primary and secondary outcomes from studies with CB and CC cardioplegia

\begin{tabular}{|c|c|c|}
\hline & $\begin{array}{c}\text { RR or WMD }[95 \% \text { CI }], P \\
\text { value }\end{array}$ & Subgroup differences \\
\hline Mortality & $\begin{array}{l}\text { CB: RR } 1.09 \text { [0.81-1.46], } \\
\quad P=.57 \\
\text { CC: RR } 0.85 \text { [0.61-1.19], } \\
\quad P=.34\end{array}$ & $\begin{array}{l}\mathrm{I}^{2}=17.4 \%, \chi^{2}=1.21 \\
\quad P=.27\end{array}$ \\
\hline MI & $\begin{array}{l}\text { CB: RR } 0.91 \text { [0.74-1.11], } \\
\quad P=.33 \\
\text { CC: RR } 1.16 \text { [0.68-1.98], } \\
\quad P=.58\end{array}$ & $\mathrm{I}^{2}=0 \%, \chi^{2}=0.75, P=.39$ \\
\hline LCOS & $\begin{array}{l}\text { CB: RR } 1.25[0.66-2.34] \\
\quad P=.49 \\
\text { CC: RR } 0.68 \text { [0.33-1.38], } \\
\quad P=.28\end{array}$ & $\begin{array}{l}\mathrm{I}^{2}=36.9 \%, \chi^{2}=1.58 \\
\quad P=.21\end{array}$ \\
\hline IABP use & $\begin{array}{l}\text { CB: RR } 1.49 \text { [0.94-2.36], } \\
\quad P=.09 \\
\text { CC: RR } 0.65 \text { [0.43-0.99], } \\
\quad P=.04\end{array}$ & $\begin{array}{l}\mathrm{I}^{2}=85.2 \%, \chi^{2}=6.78 \\
\quad P=.009\end{array}$ \\
\hline Stroke & $\begin{array}{l}\text { CB: RR } 0.96 \text { [0.64-1.45], } \\
\quad P=.86 \\
\text { CC: RR } 1.95 \text { [0.97-3.93], } \\
\quad P=.06\end{array}$ & $\begin{array}{l}\mathrm{I}^{2}=65.5 \%, \chi^{2}=2.90 \\
\quad P=.09\end{array}$ \\
\hline New AF & $\begin{array}{l}\text { CB: RR } 1.02[0.87-1.21] \\
\quad P=.78 \\
\text { CC: RR } 1.16[0.79-1.71] \\
\quad P=.45\end{array}$ & $\mathrm{I}^{2}=0 \%, \chi^{2}=0.34, P=.56$ \\
\hline AKI & $\begin{array}{l}\text { CB: RR } 0.74 \text { [0.43-1.26], } \\
\quad P=.26 \\
\text { CC: RR } 1.92 \text { [0.76-4.87], } \\
\quad P=.17\end{array}$ & $\begin{array}{l}\mathrm{I}^{2}=67.5 \%, \chi^{2}=3.08 \\
\quad P=.08\end{array}$ \\
\hline Hospital LOS & $\begin{array}{l}\text { CB: WMD }-0.62[-1.89, \\
0.64], P=.33 \\
\text { CC: WMD }-0.58[-1.44, \\
0.27], P=.18\end{array}$ & $\mathrm{I}^{2}=0 \%, \chi^{2}=0.00, P=.96$ \\
\hline
\end{tabular}


RR or WMD $[95 \% \mathrm{CI}], \boldsymbol{P}$

value

Subgroup differences

ICU LOS CB: WMD $-0.06[-1.33$,

1.21], $P=.93$

CC: WMD $-0.16[-0.46$,

$0.14], P=.30$

$C B$, Cold blood; $C C$, cold crystalloid; $R R$, relative risk; $W M D$, weighted mean difference; $C I$, confidence interval; $M I$, myocardial infarction; $L C O S$, low cardiac output syndrome $I A B P$, intra-aortic balloon pump; $A F$, atrial fibrillation; $A K I$, acute kidney injury; $L O S$, length of stay; $I C U$, intensive care unit. 